\title{
Flow- and temperature-based statistics characterizing the regimes in rapidly rotating turbulent convection in simulations employing no-slip boundary conditions
}

\author{
Citation for published version (APA): \\ Aguirre Guzmán, A. J., Madonia, M., Cheng, J. S., Ostilla-Mónico, R., Clercx, H. J. H., \& Kunnen, R. P. J. \\ (2022). Flow- and temperature-based statistics characterizing the regimes in rapidly rotating turbulent convection \\ in simulations employing no-slip boundary conditions. Physical Review Fluids, 7(1), [013501]. \\ https://doi.org/10.1103/PhysRevFluids.7.013501
}

DOI:

10.1103/PhysRevFluids.7.013501

Document status and date:

Published: 01/01/2022

\section{Document Version:}

Publisher's PDF, also known as Version of Record (includes final page, issue and volume numbers)

\section{Please check the document version of this publication:}

- A submitted manuscript is the version of the article upon submission and before peer-review. There can be important differences between the submitted version and the official published version of record. People interested in the research are advised to contact the author for the final version of the publication, or visit the DOI to the publisher's website.

- The final author version and the galley proof are versions of the publication after peer review.

- The final published version features the final layout of the paper including the volume, issue and page numbers.

Link to publication

\footnotetext{
General rights

- You may freely distribute the URL identifying the publication in the public portal. follow below link for the End User Agreement:

www.tue.nl/taverne

\section{Take down policy}

If you believe that this document breaches copyright please contact us at:

openaccess@tue.nl

providing details and we will investigate your claim.
}

Copyright and moral rights for the publications made accessible in the public portal are retained by the authors and/or other copyright owners and it is a condition of accessing publications that users recognise and abide by the legal requirements associated with these rights.

- Users may download and print one copy of any publication from the public portal for the purpose of private study or research.

- You may not further distribute the material or use it for any profit-making activity or commercial gain

If the publication is distributed under the terms of Article $25 \mathrm{fa}$ of the Dutch Copyright Act, indicated by the "Taverne" license above, please 


\title{
Flow- and temperature-based statistics characterizing the regimes in rapidly rotating turbulent convection in simulations employing no-slip boundary conditions
}

\author{
Andrés J. Aguirre Guzmán, ${ }^{1}$ Matteo Madonia ${ }^{1},{ }^{1}$ Jonathan S. Cheng, ${ }^{1, *}$ \\ Rodolfo Ostilla-Mónico ${ }^{\circ},{ }^{2}$ Herman J. H. Clercx $\odot,{ }^{1}$ and Rudie P. J. Kunnen ${ }^{1, \uparrow}$ \\ ${ }^{1}$ Fluids and Flows Group, Department of Applied Physics and J. M. Burgers Centre for Fluid Dynamics, \\ Eindhoven University of Technology, P.O. Box 513, 5600 MB Eindhoven, The Netherlands \\ ${ }^{2}$ Department of Mechanical Engineering, University of Houston, Houston, Texas 77004, USA
}

(Received 16 August 2021; accepted 9 December 2021; published 20 January 2022)

We numerically investigate flow regimes of rotating Rayleigh-Bénard convection on a horizontally periodic domain vertically bounded by no-slip walls from a statistical viewpoint. The flow is subject to strong thermal forcing [Rayleigh number up to $\mathrm{Ra}=O\left(10^{12}\right)$ ] and rapid rotation [Ekman number down to $\mathrm{Ek}=O\left(10^{-7}\right)$ ]. Various Prandtl numbers $(\mathrm{Pr}=$ $0.1, \approx 5$, and 100) are considered. In the explored parameter space, we observe the regimes of quasisteady cells, convective Taylor columns, plumes, large-scale vortices (LSVs), and rotation-affected convection. Time- and horizontally averaged statistics such as mean temperature, root-mean-square (RMS) temperature, and RMS velocity are used to discuss the thermal mixing and convective heat transfer (Nusselt number $\mathrm{Nu}$ ) in each regime. For cells and columns, both the mean temperature gradient at midheight $\left(-\left.\partial_{z}\langle T\rangle\right|_{z=0.5}\right)$ and $\mathrm{Nu}$ exhibit scalings with flow supercriticality that are consistent with predictions from numerical models of asymptotically rapidly rotating convection. For LSVs at moderate supercriticality, $-\left.\partial_{z}\langle T\rangle\right|_{z=0.5}$ saturates, akin to what is observed in geostrophic turbulence in asymptotic studies. Interestingly, for LSVs at larger supercriticality, $-\left.\partial_{z}\langle T\rangle\right|_{z=0.5}$ decreases, a behavior that is instead in line with the recently experimentally observed regime of so-called rotationally influenced turbulence. We also explore flow statistics near the top and bottom walls. The statistical distribution of temperature at the edge of the kinetic boundary layer (BL) depends on the relative thickness of this layer $\left(\delta_{u}\right)$ and the thermal BL $\left(\delta_{\theta}\right)$. In cases where $\delta_{u}>\delta_{\theta}$, the temperature distribution at $z=\delta_{u}$ is positively skewed, suggesting coherent localization of hot rising fluid; the distribution is negatively skewed at $z=1-\delta_{u}$, denoting localized cold sinking fluid. In contrast, when $\delta_{u}<\delta_{\theta}$, hot fluid at $z=\delta_{u}$ (cold fluid at $z=1-\delta_{u}$ ) occupies large portions of the horizontal domain, whereas the cold (hot) fluid is localized. Our results suggest that this near-wall statistical distribution of temperature structures, and thus the relative thickness of the kinetic and thermal BLs, does not influence the statistics in the bulk flow state. The reported findings broaden our knowledge of rotating turbulent convection at rather extreme values of the governing parameters and bounded by experimentally realizable boundary conditions, relevant to geo- and astrophysical settings.

DOI: 10.1103/PhysRevFluids.7.013501

\footnotetext{
*Present address: Department of Mechanical Engineering, University of Rochester, Rochester, New York 14627, USA.

†r.p.j.kunnen@tue.nl
} 

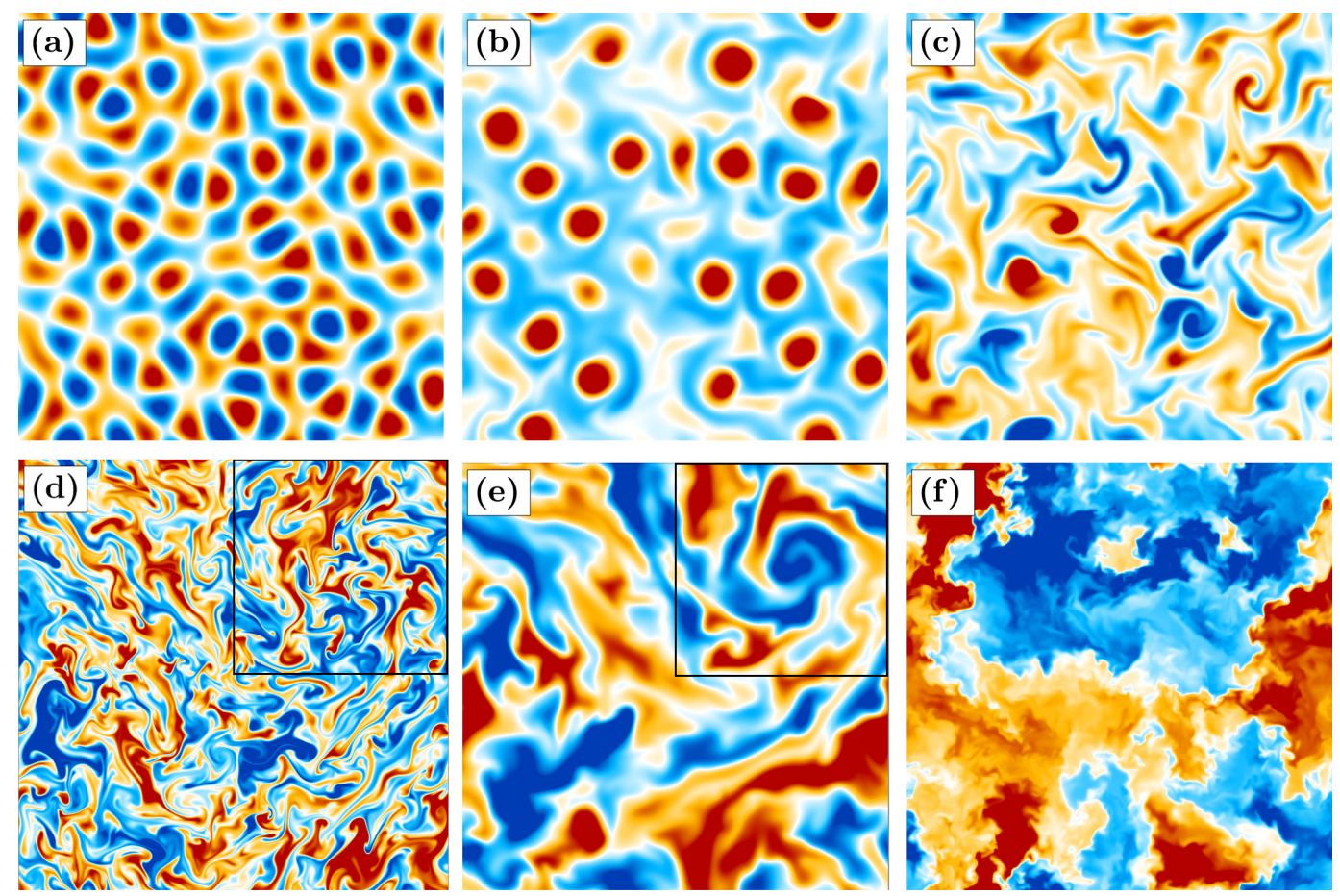

FIG. 1. Instantaneous horizontal cross sections of temperature fluctuations at midheight for selected cases at $\operatorname{Pr} \approx 5$ in the regimes of (a) cells $\left(\mathrm{Ra} / \mathrm{Ra}_{c}=1.3\right)$, (b) convective Taylor columns $\left(\mathrm{Ra} / \mathrm{Ra} a_{c}=3.5\right)$, (c) plumes $\left(\mathrm{Ra} / \mathrm{Ra}_{c}=7.5\right)$, and (d) large-scale vortices $\left(\mathrm{Ra} / \mathrm{Ra}_{c} \approx 80\right)$, and at $\operatorname{Pr}=0.1$ in the regimes of (e) large-scale vortices $\left(\mathrm{Ra} / \mathrm{Ra}_{c} \approx 20\right)$, and (f) rotation-affected convection $\left(\mathrm{Ra} / \mathrm{Ra}_{c} \approx 489\right)$. The color scale is chosen to highlight the flow features. Red and blue denote above- and below-average temperature fluctuations, respectively. The domain-size vortical flows in panels (d) and (e) lead to large swirling patterns of temperature fluctuations; the cyclonic structure therein is indicated with a thin-lined box. The observed flow regimes are identified based on their flow- and temperature-based statistical properties (discussed in the present paper), their characteristic force balance (as we report in Ref. [17]), and their scale-by-scale kinetic energy transfer (as we discuss in Ref. [18]).

\section{INTRODUCTION}

Rotating Rayleigh-Bénard convection (RRBC), the buoyancy-driven flow between two parallel horizontal plates and under the influence of rotation, provides a simplified framework to study numerous large-scale flows in nature. Planetary atmospheres, terrestrial oceans, and stellar and planetary interiors are prominent scenarios where fluid motion arises due to buoyancy forcing and is altered by rotation of the celestial body (see, e.g., Refs. [1-4]). Hence a thorough understanding of the flow dynamics in the RRBC configuration holds great importance in geophysics and astrophysics. The current literature of rapidly rotating convection discerns a wealth of rotating convection regimes when the strength of the buoyancy forcing and rotation are varied; see Ref. [5] for a recent review. These are regimes of cellular convection, convective Taylor columns, plumes, geostrophic turbulence [where domain-filling large-scale vortices (LSVs) may be observed], rotation-affected convection, and rotation-unaffected convection. The flow in these regimes, except for rotationunaffected convection, is visualized through temperature fluctuations in Fig. 1. Much of the characterization of these flow states involves the assessment of flow- and temperature-based statistical quantities. This is, on the one hand, due to their accessibility in laboratory experiments of rotating convection [6-13] and, on the other hand, because they can be used to identify transitions 
between the flow regimes [10,13-15]. For instance, through simulations of reduced equations in the asymptotic limit of rapid rotation in Ref. [14], it has been shown that the time- and plane-averaged vertical profile of temperature $\langle T\rangle(z)$, often characterized by its gradient at midheight $-\left.\partial_{z}\langle T\rangle\right|_{z=0.5}$, varies nonmonotonically with flow supercriticality, granting it diagnostic properties of the various flow phenomenologies. Here we quantify flow supercriticality as $\mathrm{Ra} / \mathrm{Ra}_{c}$, the ratio of the Rayleigh number $\mathrm{Ra}$ and its critical value for onset of convection $\mathrm{Ra}_{c}$ (both defined below in Sec. II). In particular, the mean temperature gradient decreases with $\mathrm{Ra} / \mathrm{Ra}_{c}$ in the cellular and columnar regimes, it then increases in the plumes regime, and saturates (becomes constant) in the geostrophic turbulence regime [14]. At larger flow supercriticality, beyond the limit of validity of asymptotic models, recent laboratory experiments in Ref. [13] have revealed that $-\left.\partial_{z}\langle T\rangle\right|_{z=0.5}$ decreases again with supercriticality, according to the scaling $-\left.\partial_{z}\langle T\rangle\right|_{z=0.5} \sim \mathrm{Ra}^{-0.21}$; the convective heat transfer (convective Nusselt number $\mathrm{Nu}$ ) scales as $\mathrm{Ra}^{0.52}$. These scaling behaviors are deemed to describe a regime of so-called rotationally influenced turbulence (RIT) [13]. Past this regime, a state of rotation-affected convection is found, where $-\left.\partial_{z}\langle T\rangle\right|_{z=0.5}$ may exhibit a steeper decrease with flow supercriticality, as suggested by the experimental data in Ref. [13]. Finally, at sufficiently large $\mathrm{Ra} / \mathrm{Ra}_{c}$, it is expected that $-\left.\partial_{z}\langle T\rangle\right|_{z=0.5} \approx 0$, as the flow becomes insensitive to rotation and the bulk becomes nearly isothermal.

In addition to the midheight mean temperature gradient, in systems bounded by no-slip top and bottom walls, the near-wall flow dynamics in itself can also be assessed to characterize the bulk phenomenology. In linear Ekman boundary layer (BL) theory [16], the thickness $\delta_{u}$ of the kinetic boundary layer that forms near the no-slip walls is established to scale as $\mathrm{Ek}^{1 / 2}$, when the distant flow is in geostrophic balance (the balance between Coriolis and pressure-gradient forces). Therefore, the scaling $\delta_{u} \sim \mathrm{Ek}^{1 / 2}$ is a fundamentally relevant indicator of rotational control in the fluid layer, and hence its compliance characterizes the known geostrophic regimes: cells, convective Taylor columns, plumes, and geostrophic turbulence. For RIT, the role of rotation is hypothesized to be important but not as dominant as in geostrophic turbulence. Hence, it remains an open question whether such dynamics can lead to the development of Ekman-type BLs in the RIT regime. In asymptotic studies in Ref. [14], additional characterization of the geostrophic regimes is attained through the root-mean-square (RMS) and kurtosis (defined in Sec. IV D) of various physical quantities, yet some of the underlying aspects of flows under realistic conditions may not exhibit asymptotic behaviors. In the asymptotic limit of rapid rotation, a symmetric state is obtained where the skewness, or asymmetry, of the statistical distribution of several physical quantities is zero over horizontal planes at all domain heights. Direct numerical simulation (DNS) performed at finite rotation rates, on the other hand, reveal great asymmetries in these distributions [19-21]. Similar findings are reported in laboratory experiments and in studies of geophysical flows (see, e.g., review in Ref. [22] and references therein). The presence of these asymmetries in natural flows, and their quantitative study, can be exploited to provide additional means for flow characterization. This is enabled in the present study of RRBC, as we employ finite rotation rates in our DNS.

In this study we discuss the most prominent differences and similarities between the flow configurations in the asymptotic limit of rapid rotation and those manifested in our setup at finite rotation rate. We address the distinction and characterization of RRBC regimes based on numerous flow- and temperature-based statistical quantities. Furthermore, we evaluate the effect of the no-slip (top and bottom) walls on the aforementioned statistical quantities, and their impact on the bulk flow. We present an investigation of the statistics in the presence of no-slip walls, providing a detailed comparison with the observed stress-free-type behavior in asymptotic studies. For the task, we perform DNS of RRBC, at various Prandtl numbers $\operatorname{Pr}(=0.1, \sim 5$, and 100), on a laterally periodic domain vertically bounded by no-slip walls. This paper provides an analysis similar to Ref. [19], but in a substantially extended parameter range to describe the geostrophic regime of RRBC (which was computationally out of reach at that time). The statistical characterization of RRBC regimes presented below can ultimately provide a framework for identification of these regimes in future laboratory experiments and numerical simulations at even more extreme parameter values, and for extrapolation to geophysical and astrophysical settings. 
The layout of the paper is as follows. The governing equations of RRBC and the associated dimensionless parameters are introduced in Sec. II. In Sec. III we describe the numerical setup and present the parameter values for the simulations. In Sec. IV A we investigate the midheight mean temperature gradient and the heat transport across the fluid layer, and compare these results with those from asymptotic studies to distinguish the different flow regimes. Next, in Sec. IV B we discuss the (scaling) characteristics of the thermal and kinetic BL thicknesses. In Sec. IV C we gain insight into the flow and temperature structures by means of the temperature, vertical-velocity, and vertical-vorticity skewness. We further characterize the flow and thermal features by evaluating the likelihood of extreme values (kurtosis) of the relevant physical quantities in Sec. IV D. Last, in Sec. V we present our conclusions.

\section{GOVERNING EQUATIONS}

We model rotating Rayleigh-Bénard convection using the Navier-Stokes equations plus an advection-diffusion equation for temperature, in the Boussinesq approximation [23]:

$$
\begin{aligned}
\frac{\partial u}{\partial t}+(u \cdot \nabla) u+\frac{1}{\operatorname{Ro}_{\mathrm{C}}} \hat{z} \times u & =-\nabla p+\sqrt{\frac{P r}{R a}} \nabla^{2} u+T \hat{z}, \\
\frac{\partial T}{\partial t}+(u \cdot \nabla) T & =\frac{1}{\sqrt{\operatorname{RaPr}}} \nabla^{2} T,
\end{aligned}
$$

with the incompressibility condition

$$
\nabla \cdot u=0
$$

Here velocity $u$, temperature $T$, pressure $p$, and time $t$ are nondimensionalized using the domain height $H$, bottom-top temperature difference $\Delta T=T_{\text {bottom }}-T_{\text {top }}>0$, and characteristic "free-fall" velocity scale $U_{\mathrm{ff}}=\sqrt{g \alpha \Delta T H}$. The rotation vector $\Omega \hat{z}$ is parallel to the vertical unit vector $\hat{z}$ ( $\Omega$ is the angular velocity), whereas the gravitational acceleration is $-g \hat{z}$. The kinematic viscosity, thermal diffusivity, and thermal expansion coefficient of the fluid are $\nu, \kappa$, and $\alpha$, respectively.

The nondimensional parameters $\operatorname{Ra}=g \alpha \Delta T H^{3} /(\nu \kappa), \operatorname{Pr}=v / \kappa$, and $\operatorname{Ro}_{\mathrm{C}}=\sqrt{g \alpha \Delta T / H} /(2 \Omega)$ are the Rayleigh number, Prandtl number, and convective Rossby number, respectively. The first one characterizes the strength of the thermal forcing, Pr involves the diffusive properties of the fluid, and $\mathrm{Ro}_{\mathrm{C}}$ parametrizes the (inverse) strength of rotation. Alternatively, rotation can be characterized through the Ekman number Ek $=v /\left(2 \Omega H^{2}\right)=\operatorname{Ro}_{C} \sqrt{\operatorname{Pr} / \operatorname{Ra}}$.

The top and bottom boundaries are impenetrable (vertical velocity $w=0$ ) and no-slip boundary conditions (horizontal velocities $u=v=0$ ) apply. For reference, a few cases with stress-free boundary conditions $(\partial u / \partial z=\partial v / \partial z=w=0)$ are considered. The boundaries are taken to be perfect thermal conductors ( $T=1$ at the bottom and $T=0$ at the top).

The critical Rayleigh number $\mathrm{Ra}_{c}$ for onset of convection in a laterally unbounded fluid layer subject to rapid rotation $\left(\mathrm{Ek} \lesssim 10^{-3}\right.$ ) [23,24] is used to define flow supercriticality as $\mathrm{Ra} / \mathrm{Ra}_{c}$. The value of $\mathrm{Ra}_{c}$ and the type of convection structures depend on the Prandtl number. At $\operatorname{Pr}<0.68$, fluid motion starts in the form of oscillatory convection at $\mathrm{Ra}_{c}=17.4(\mathrm{Ek} / \mathrm{Pr})^{-4 / 3}$. At $\operatorname{Pr} \geqslant 0.68$, convection sets in as steady cells at $\mathrm{Ra}_{c}=8.7 \mathrm{Ek}^{-4 / 3}$. Throughout this work, we present our results in terms of $\mathrm{Ra} / \mathrm{Ra}_{c}$. The characteristic horizontal length scale $\ell_{c}$ of the convection structures also depends on $\operatorname{Pr}: \ell_{c}=2.4(\mathrm{Ek} / \operatorname{Pr})^{1 / 3}$ at $\operatorname{Pr}<0.68$ and $\ell_{c}=2.4 \mathrm{Ek}^{1 / 3}$ at $\operatorname{Pr} \geqslant 0.68$ [23,25,26], again, valid for a laterally unbounded layer of fluid subject to rapid rotation $\left(\mathrm{Ek} \lesssim 10^{-3}\right)$.

\section{NUMERICAL METHODOLOGY}

We solve Eqs. (1) to (3) on a laterally periodic Cartesian domain, using two codes: a single-grid code, where both velocity and temperature are resolved on the same grid, and a multiple-grid code, where velocity is resolved on a coarser grid than that for temperature. The former is used for cases at low $\operatorname{Pr}<1$, where the smallest active length scale for velocity (Kolmogorov length scale) is smaller 
TABLE I. Model parameters used in the DNS. Prandtl number Pr, Ekman number Ek, Rayleigh number $\mathrm{Ra}$, convective Rossby number $\mathrm{Ro}_{\mathrm{C}}$, supercriticality $\mathrm{Ra} / \mathrm{Ra}_{c}$, and width-to-height domain aspect ratio $\Gamma$. In the simulation series at $\operatorname{Pr} \approx 5$, the small difference in both $\mathrm{Pr}$ and $\mathrm{Ek}$ is for comparison with experiments in our group [13,31,32]. The number of grid points to resolve the velocity field is $N_{x}, N_{y}$, and $N_{z}$, and the refinement factors for temperature $m_{x}, m_{y}$, and $m_{z}$ (used by the multiple-grid code). The last column indicates the observed flow structures: convective cells (C), convective Taylor columns (T), plumes (P), large-scale vortices (LSVs), or rotation-affected convection (RA). All cases are simulated with no-slip walls. Cases denoted with an asterisk superscript are also independently simulated with stress-free boundaries. The case denoted with a dagger presents signs of upscale energy transfer, but no LSVs develop.

\begin{tabular}{|c|c|c|c|c|c|c|c|c|}
\hline $\operatorname{Pr}$ & Ek & $\mathrm{Ra}$ & $\operatorname{Ro}_{\mathrm{C}}$ & $\mathrm{Ra} / \mathrm{Ra}_{c}$ & $\Gamma$ & $N_{x} \times N_{y} \times N_{z}$ & $m_{x} \times m_{y} \times m_{z}$ & Flow \\
\hline $\operatorname{Pr}$ & $\mathrm{Ek}$ & $\mathrm{Ra}$ & $\operatorname{Ro}_{\mathrm{C}}$ & $\mathrm{Ra} / \mathrm{Ra}_{c}$ & $\Gamma$ & $N_{x} \times N_{y} \times N_{z}$ & $m_{x} \times m_{y} \times m_{z}$ & Flow \\
\hline 0.1 & $2.00 \times 10^{-7}$ & $1.00 \times 10^{10}$ & 0.063 & 14.48 & 0.302 & $1024 \times 1024 \times 1408$ & - & LSV \\
\hline 0.1 & $2.24 \times 10^{-7}$ & $1.00 \times 10^{10}$ & 0.071 & 16.84 & 0.314 & $1024 \times 1024 \times 1408$ & - & LSV \\
\hline 0.1 & $2.50 \times 10^{-7}$ & $1.00 \times 10^{10}$ & 0.079 & 19.50 & 0.326 & $768 \times 768 \times 1280$ & - & $\mathrm{LSV}^{*}$ \\
\hline 0.1 & $3.00 \times 10^{-7}$ & $1.00 \times 10^{10}$ & 0.095 & 24.87 & 0.346 & $768 \times 768 \times 1280$ & - & LSV \\
\hline 0.1 & $4.00 \times 10^{-7}$ & $1.00 \times 10^{10}$ & 0.126 & 36.49 & 0.381 & $640 \times 640 \times 1280$ & - & LSV \\
\hline 0.1 & $8.00 \times 10^{-7}$ & $1.00 \times 10^{10}$ & 0.253 & 91.95 & 0.480 & $640 \times 640 \times 1280$ & - & RA \\
\hline 0.1 & $1.05 \times 10^{-6}$ & $1.00 \times 10^{10}$ & 0.332 & 132.14 & 0.526 & $768 \times 768 \times 1280$ & - & RA \\
\hline 0.1 & $2.80 \times 10^{-6}$ & $1.00 \times 10^{10}$ & 0.885 & 488.65 & 0.729 & $1088 \times 1088 \times 1280$ & - & RA \\
\hline 0.1 & $6.00 \times 10^{-6}$ & $1.00 \times 10^{10}$ & 1.897 & 1349.95 & 0.940 & $1408 \times 1408 \times 1280$ & - & RA \\
\hline 5.5 & $3.00 \times 10^{-7}$ & $5.50 \times 10^{9}$ & 0.009 & 1.27 & 0.323 & $256 \times 256 \times 640$ & $2 \times 2 \times 1$ & $\mathrm{C}$ \\
\hline 5.5 & $3.00 \times 10^{-7}$ & $8.00 \times 10^{10}$ & 0.011 & 1.85 & 0.323 & $256 \times 256 \times 640$ & $2 \times 2 \times 1$ & $\mathrm{C}$ \\
\hline 5.5 & $3.00 \times 10^{-7}$ & $1.00 \times 10^{10}$ & 0.013 & 2.31 & 0.323 & $384 \times 384 \times 640$ & $2 \times 2 \times 1$ & $\mathrm{~T}$ \\
\hline 5.5 & $3.00 \times 10^{-7}$ & $1.50 \times 10^{10}$ & 0.016 & 3.46 & 0.323 & $384 \times 384 \times 640$ & $2 \times 2 \times 1$ & $\mathrm{~T}$ \\
\hline 5.5 & $3.00 \times 10^{-7}$ & $2.00 \times 10^{10}$ & 0.018 & 4.62 & 0.323 & $384 \times 384 \times 640$ & $2 \times 2 \times 1$ & $\mathrm{~T}$ \\
\hline 5.2 & $1.00 \times 10^{-7}$ & $1.40 \times 10^{11}$ & 0.016 & 7.47 & 0.224 & $384 \times 384 \times 640$ & $2 \times 2 \times 2$ & $\mathrm{P}^{*}$ \\
\hline 5.2 & $1.00 \times 10^{-7}$ & $2.10 \times 10^{11}$ & 0.020 & 11.20 & 0.224 & $384 \times 384 \times 640$ & $2 \times 2 \times 2$ & $\mathrm{P}$ \\
\hline 5.2 & $1.00 \times 10^{-7}$ & $3.20 \times 10^{11}$ & 0.025 & 17.07 & 0.224 & $512 \times 512 \times 640$ & $2 \times 2 \times 2$ & $\mathrm{P}^{*}$ \\
\hline 5.2 & $1.00 \times 10^{-7}$ & $6.00 \times 10^{11}$ & 0.034 & 32.01 & 0.224 & $512 \times 512 \times 640$ & $2 \times 2 \times 2$ & $\mathrm{P}^{\dagger}$ \\
\hline 5.2 & $1.00 \times 10^{-7}$ & $9.50 \times 10^{11}$ & 0.043 & 50.68 & 0.224 & $640 \times 640 \times 896$ & $2 \times 2 \times 2$ & LSV \\
\hline 5.2 & $1.00 \times 10^{-7}$ & $1.50 \times 10^{12}$ & 0.054 & 80.03 & 0.224 & $768 \times 768 \times 1024$ & $2 \times 2 \times 2$ & $\mathrm{LSV}^{*}$ \\
\hline 100 & $3.00 \times 10^{-7}$ & $1.30 \times 10^{11}$ & 0.011 & 30.01 & 0.323 & $384 \times 384 \times 512$ & $3 \times 3 \times 3$ & $\mathrm{P}$ \\
\hline 100 & $3.00 \times 10^{-7}$ & $2.10 \times 10^{11}$ & 0.014 & 48.48 & 0.323 & $384 \times 384 \times 512$ & $3 \times 3 \times 3$ & $\mathrm{P}$ \\
\hline 100 & $3.00 \times 10^{-7}$ & $3.40 \times 10^{11}$ & 0.017 & 78.49 & 0.323 & $512 \times 512 \times 512$ & $3 \times 3 \times 3$ & $\mathrm{P}$ \\
\hline 100 & $3.00 \times 10^{-7}$ & $6.00 \times 10^{11}$ & 0.023 & 138.50 & 0.323 & $512 \times 512 \times 768$ & $3 \times 3 \times 3$ & $\mathrm{P}$ \\
\hline 100 & $3.00 \times 10^{-7}$ & $9.50 \times 10^{11}$ & 0.029 & 219.30 & 0.323 & $512 \times 512 \times 768$ & $3 \times 3 \times 3$ & $\mathrm{P}$ \\
\hline 100 & $3.00 \times 10^{-7}$ & $1.50 \times 10^{12}$ & 0.037 & 346.26 & 0.323 & $512 \times 512 \times 768$ & $3 \times 3 \times 3$ & $\mathrm{P}$ \\
\hline 100 & $3.00 \times 10^{-7}$ & $2.50 \times 10^{12}$ & 0.047 & 577.10 & 0.323 & $384 \times 384 \times 768$ & $4 \times 4 \times 4$ & $\mathrm{P}$ \\
\hline
\end{tabular}

than that for temperature (Batchelor length scale), whereas the latter code is advantageous for cases at high $\operatorname{Pr}>1$, where the Batchelor length scale is smaller. Both codes are based on the Verzicco convection code [27,28]. For additional details on the codes and their validation, see Refs. [27-30]. A list of the cases considered in this study is presented in Table I. Notice that the set of simulations at $\operatorname{Pr} \approx 5$ comprises a small difference in both Pr and Ek that is for comparison with (ongoing) laboratory experiments in our group $[13,31,32]$.

The domain size, $10 \ell_{c} \times 10 \ell_{c} \times 1$ (normalized by the domain height $H$ ) at low Pr, and $20 \ell_{c} \times 20 \ell_{c} \times 1$ at high $\mathrm{Pr}$, lead to appropriate convergence of spatially averaged statistics. For all runs, the largest grid spacing in the bulk is below four times the smallest active length scale (either Kolmogorov or Batchelor length scale), which is considered adequate in Ref. [28]. In both codes, the grid is vertically denser near the top and bottom to appropriately resolve the thinner (kinetic or 
thermal) boundary layer. We find, in postprocessing, that a minimum of 11 grid points are allocated within this region, which is enough to accurately resolve it.

The measure of the convective heat transfer across the fluid layer is used to further validate the grid resolution: we compute $\mathrm{Nu}$ in five different ways (as the plane-averaged wall-normal temperature gradient at the bottom and at the top wall, as the volume-averaged convective flux, and based on exact relations for the dissipation rate of kinetic energy and for that of thermal variance [33]) and confirm the convergence of these measurements to within a few percent, over simulation times of the order of $10^{2}$ convective time units $\tau_{\mathrm{ff}}=H / U_{\mathrm{ff}}$.

\section{RESULTS}

\section{A. Temperature gradient and heat transport}

A well-known feature of rotating Rayleigh-Bénard convection is the gradual decrease of the mean bulk temperature with height across the fluid layer [13,19,21,34], from a large value at the hot bottom wall to a lower temperature at the top (this is also observed, e.g., in nonrotating RBC at low $\operatorname{Pr} \leqslant 1[35,36])$. As a function of $\mathrm{Ra} / \mathrm{Ra}_{c}$, the vertical profile of mean temperature can be evaluated in two limit cases. At $\mathrm{Ra} / \mathrm{Ra}_{c}<1$, where no convection is present, the heat transfer from the bottom wall to the top wall is carried out by conduction alone. In this state the temperature profile is linear and the midheight mean temperature gradient is maximal, $-\left.\partial_{z}\langle T\rangle\right|_{z=0.5}=1$. In the limit of very large $\mathrm{Ra} / \mathrm{Ra}_{c}$, the influence of rotation is minimal, and the flow approaches a nonrotating style of convection. In this state the bulk is well mixed, so that its mean temperature is uniform, and $-\left.\partial_{z}\langle T\rangle\right|_{z=0.5} \approx 0$ (in the bulk outside the BLs). For values of $\mathrm{Ra} / \mathrm{Ra}_{c}$ between these two extreme cases, the temperature gradient exhibits variations that are specific to the distinct flow regimes, and thus provide a way to characterize them.

Figure 2(a) shows the mean temperature gradient at midheight, $-\left.\partial_{z}\langle T\rangle\right|_{z=0.5}$, as a function of $\mathrm{Ra} / \mathrm{Ra}_{c}$ for all our simulation cases at all Prandtl numbers explored. At $\mathrm{Pr} \approx 5$, we observe that the temperature gradient decreases with supercriticality throughout the cellular and columnar regimes (also reported in Refs. [13,14]). Here $-\left.\partial_{z}\langle T\rangle\right|_{z=0.5}$ scales as $\left(\mathrm{Ra} / \mathrm{Ra}_{c}\right)^{-0.91 \pm 0.07}$, which is in good agreement with the asymptotic scaling $-\left.\partial_{z}\langle T\rangle\right|_{z=0.5} \sim\left(\operatorname{RaEk}^{4 / 3}\right)^{-0.96} \sim\left(\mathrm{Ra} / \mathrm{Ra}_{c}\right)^{-0.96}$ in Ref. [14] [see dotted line in Fig. 2(a)]. This drop in temperature gradient is accompanied by an increase in heat transport [see Fig. 2(b)]. The Nusselt number data corresponding to the four cases at $\operatorname{Pr} \approx 5$ and lowest $\mathrm{Ra} / \mathrm{Ra}_{c}$ describe a scaling behavior that is steeper than that provided by the asymptotic exponent $\gamma=2.1$ [black dotted line in Fig. 2(b)]for cells and columns with stress-free boundary conditions [14]. Our data are in fact in better agreement with $\gamma=3$ obtained through asymptotic models with parametrized Ekman pumping, and DNS with no-slip walls, in Ref. [37]. Surely, the increased heat transport efficiency (i.e., larger scaling exponent) is associated with Ekman pumping from the kinetic boundary layers [15,37,44-46], which is absent when stress-free boundary conditions are considered, as in asymptotic studies in Ref. [14]. The enhancement in heat transport is most clearly visible for cellular and columnar structures, where the Ekman flow at both boundaries increases the heat transport through these vertically aligned structures.

In the plumes regime, $-\left.\partial_{z}\langle T\rangle\right|_{z=0.5}$ increases with $\mathrm{Ra} / \mathrm{Ra}_{c}$ (also for plumes at $\mathrm{Pr}=100$ ), as observed in Refs. $[13,14]$. Here vertical heat transport is hindered by increasingly strong horizontal advection due to ever stronger interaction among plumes. Hence, the increase of $\mathrm{Nu}$ with $\mathrm{Ra} / \mathrm{Ra}{ }_{c}$ becomes shallower. A least-squares fit of the Nusselt number for plumes at $\operatorname{Pr} \approx 5$ [i.e., for $6 \leqslant$ $\mathrm{Ra} / \mathrm{Ra}_{c} \leqslant 37$ in Fig. 2(b)] yields a scaling $\mathrm{Nu} \sim\left(\mathrm{Ra} / \mathrm{Ra}_{c}\right)^{0.42 \pm 0.03}$, a reduction of about $85 \%$ in the scaling exponent with respect to the cellular and columnar regimes. For plumes at $\operatorname{Pr}=100$, the scaling is $\mathrm{Nu} \sim\left(\mathrm{Ra} / \mathrm{Ra}_{c}\right)^{0.24 \pm 0.01}$.

In the LSV regime at $\mathrm{Ra} / \mathrm{Ra}_{c}>37$ (at $\mathrm{Pr} \approx 5$ ), the temperature gradient gradually reverses back to a downward trend. This behavior differs from what is observed in the geostrophic turbulence regime, which is characterized by a saturation of the midheight mean temperature gradient (i.e., $-\left.\partial_{z}\langle T\rangle\right|_{z=0.5}$ does not change with $\mathrm{Ra} / \mathrm{Ra}_{c}$ ) [14]. The decreasing behavior in our data for 

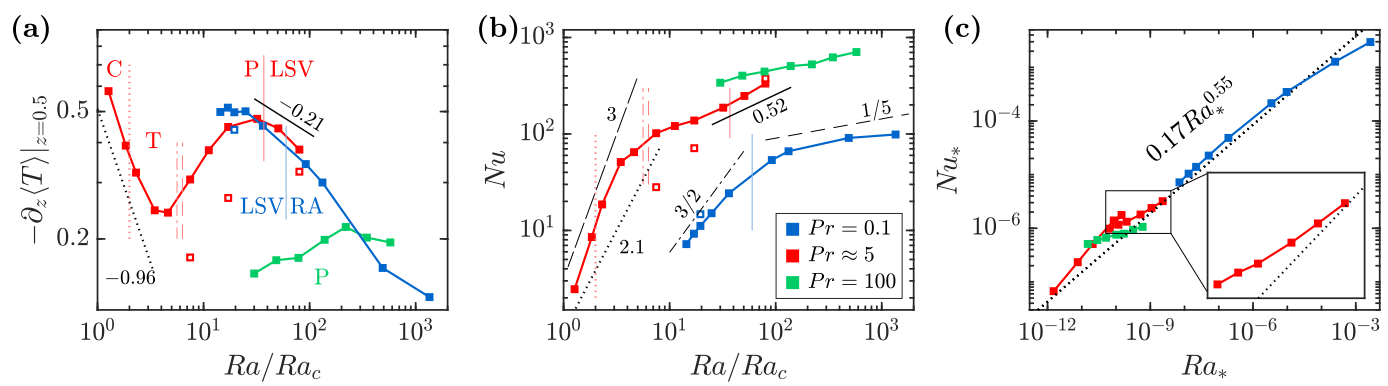

FIG. 2. (a) Midheight mean temperature gradient at various Prandtl numbers [indicated in the legend of panel (b)]; the black dotted line is the asymptotic scaling $\sim\left(\mathrm{Ra} / \mathrm{Ra}_{c}\right)^{-0.96}$ for cells and columns [14]; the black line is the scaling $\sim\left(\mathrm{Ra} / \mathrm{Ra}_{c}\right)^{-0.21}$ for so-called rotation-influenced turbulence (RIT; in water experiments at $\operatorname{Pr} \approx 5.2$ ) [13]. These and other scalings are indicated through their scaling exponent (i.e., the number next to a given scaling). (b) Convective Nusselt number $\mathrm{Nu}$; the black dotted line is the asymptotic scaling for cells and columns; the black long-dashed line is the scaling from asymptotic models with parametrized Ekman pumping and DNS with no-slip walls in Ref. [37]; the black (solid) line is the scaling for RIT [13]; the black dash-dotted line is the asymptotic scaling in the geostrophic turbulence regime; the black short-dashed line is the predicted scaling for nonrotating RBC (Ek is taken as a constant) at $\operatorname{Pr}=0.1$ [38-41]. In both panels (a) and (b), vertical dotted lines are our qualitative estimate for the transition between cells (C) and convective Taylor columns (T). Vertical dash-dotted and dashed lines are predicted transitions between $T$ and plumes $(\mathrm{P})$ in Refs. [10] and [42], respectively. Vertical solid lines are our qualitative estimate for the transitions between $P$ and large-scale vortices (LSVs) at $P r \approx 5$ (in red), and between LSVs and rotation-affected (RA) convection at $\operatorname{Pr}=0.1$ (in blue). (c) $\mathrm{Nu}_{*} \mathrm{vs} \mathrm{Ra}_{*}$ (both defined in the text); the dotted line is the scaling $\mathrm{Nu}_{*}=0.17 \mathrm{Ra}_{*}^{0.55}$ in Ref. [43]. The inset zooms in on cases at $\operatorname{Pr}=5.2$ (and $\mathrm{Ek}=10^{-7}$ ) in the intervals $5 \times 10^{-11} \leqslant \mathrm{Ra}_{*} \leqslant 4 \times 10^{-9}$ and $8 \times 10^{-7} \leqslant \mathrm{Nu}_{*} \leqslant 5 \times 10^{-6}$. In all panels filled and open symbols are for no-slip and stress-free simulations, respectively.

$-\left.\partial_{z}\langle T\rangle\right|_{z=0.5}$ is instead consistent with that reported in the RIT regime [13]. In fact, our data (also at $\operatorname{Pr}=0.1$, discussed below) appears to be well described by the proposed scaling $-\left.\partial_{z}\langle T\rangle\right|_{z=0.5} \sim$ $\left(\mathrm{Ra} / \mathrm{Ra}_{c}\right)^{-0.21}$ (at fixed Ek) in this regime [compare the last three red data points with the solid black line in Fig. 2(a)]. Below we discuss these observations further. In our LSV regime (at $\operatorname{Pr} \approx 5$ ), vertical-velocity fluctuations again increase with supercriticality. This increase in $w_{\text {RMS }}$ is rather moderate, yet enough to enhance vertical mixing, leading to a Nu scaling that appears to be slightly steeper than the scaling $\left(\mathrm{Ra} / \mathrm{Ra}_{c}\right)^{0.42 \pm 0.03}$ obtained in the plumes regime. While our data are scarce, our cases may be consistent with the slightly steeper scaling $\left(\mathrm{Ra} / \mathrm{Ra}_{c}\right)^{0.52}$ for RIT [black solid line in Fig. 2(b)], as proposed in Ref. [13]. To further evaluate the Nu scaling, we consider the scaling law in Ref. [43]: $\mathrm{Nu}_{*}=a \mathrm{Ra}_{*}^{b}$, where $\mathrm{Nu}_{*}=\mathrm{NuEk} / \mathrm{Pr}$ and $\mathrm{Ra}_{*}=\mathrm{RaEk}^{3} \mathrm{Nu} / \mathrm{Pr}^{2}$, with best-fit parameters $a=0.17$ and $b=0.55$. Here the prefactor $a$ has been multiplied by $2^{3 b-1}$ to account for the factor of 2 in the different definitions of Ek used in Ref. [43] and in this paper. This scaling law is independent of the diffusive properties (thermal diffusivity $\kappa$ and kinematic viscosity $v$ ) of the fluid. While the presence of LSVs in the simulations in Ref. [43] is not mentioned, this scaling is proposed for a rotation-dominated flow, at $\operatorname{Pr}=0.7$ and 7 , subject to competing nonlinear effects, in the presence of stress-free walls. In Ref. [47], $\mathrm{Nu}_{*}=0.17 \mathrm{Ra}_{*}^{0.55}$ is shown to fit fairly well Nu measurements of LSV cases at $\mathrm{Pr}=1$. In Fig. 2(c) we plot $\mathrm{Nu}_{*}$ as a function of $\mathrm{Ra}_{*}$ for all our simulation cases. In the close-up of the $\operatorname{Pr} \approx 5$ data, shown in the inset, we notice that the data appear to approach the proposed scaling.

In the LSV regime at $\operatorname{Pr}=0.1$ (for $\mathrm{Ra} / \mathrm{Ra}_{c}<60$ ), the midheight mean temperature gradient displays little variation with $\mathrm{Ra} / \mathrm{Ra}_{c}$ for the four cases with lowest supercriticality. This is consistent with the saturation in $-\left.\partial_{z}\langle T\rangle\right|_{z=0.5}$ that characterizes the geostrophic turbulence regime [14]. Nonetheless, $-\left.\partial_{z}\langle T\rangle\right|_{z=0.5}$ is smaller for the fifth case at $\mathrm{Ra} / \mathrm{Ra}_{c} \approx 36$, suggesting that, also at this Prandtl number, LSVs can occur in the range where $-\left.\partial_{z}\langle T\rangle\right|_{z=0.5}$ is on a downward trend. Therefore, 
(a)

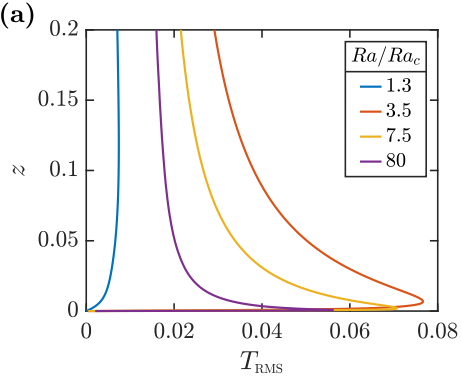

(b)

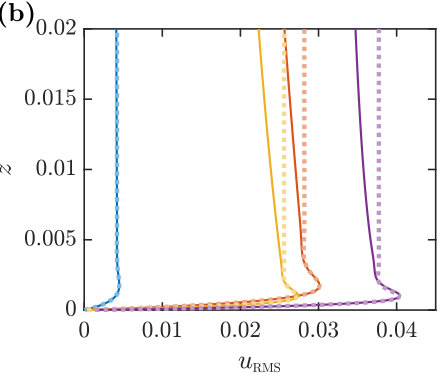

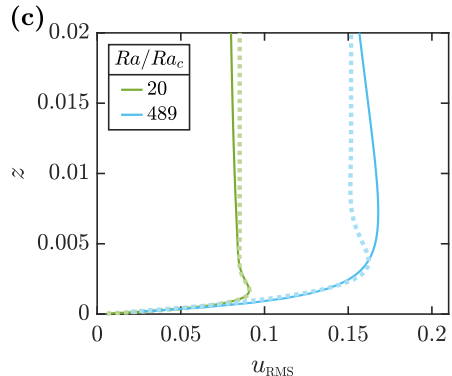

FIG. 3. Vertical profiles of (a) RMS temperature and (b) RMS horizontal velocity, for selected cases at $\operatorname{Pr} \approx 5$, and (c) RMS horizontal velocity for selected cases at $\operatorname{Pr}=0.1$ displaying LSVs $\left(\mathrm{Ra} / \mathrm{Ra}_{c} \approx 20\right)$ and rotation-affected convection $\left(\mathrm{Ra} / \mathrm{Ra}_{c} \approx 489\right)$. The profiles, which are symmetric about $z=0.5$, are shown near the bottom wall of the domain. Dotted lines in panels (b) and (c) are the theoretical Ekman BL profile (see text for analytical solution) fitted to each $u_{\mathrm{RMS}}(z)$ profile in the range $0<z<3 \times 10^{-3}$ [for the case at $\left(\mathrm{Pr}, \mathrm{Ra} / \mathrm{Ra}_{c}\right)=(0.1,489)$ in panel $(\mathrm{c})$, we use $0<z<5 \times 10^{-2}$ to take into account the thicker BL region].

our LSV cases at $\operatorname{Pr}=0.1$ and lowest $\mathrm{Ra} / \mathrm{Ra}_{c}$ can be regarded as part of the geostrophic turbulence regime, whereas those at larger $\mathrm{Ra} / \mathrm{Ra}_{c}$ are expected to be part of the RIT regime, as observed at $\operatorname{Pr} \approx 5$. Hence, our results indicate the presence of LSVs beyond the geostrophic turbulence regime, uncovering their existence within the RIT regime. Notably, LSV-dominant convection has been previously observed in the "plumes" regime of asymptotically rapidly rotating convection [48]. Our results thus provide additional evidence that such dynamics is indeed not exclusive to the geostrophic turbulence regime. At this point, we would like to also highlight the following: (1) In Ref. [14], it is explained that access to geostrophic turbulence is more readily gained at lower $\operatorname{Pr}$; this is confirmed here not only through comparison of our data at $\operatorname{Pr}=0.1$ and at $\operatorname{Pr} \approx 5$, but also by noting that flows at $\operatorname{Pr}=100$ would demand significantly higher $\mathrm{Ra} / \mathrm{Ra}_{c}$ to achieve this flow state. (2) The presence of LSVs in the RIT regime certainly suggests that rotation exerts a substantial influence in this regime. We shall further discuss the strength of these rotational effects in Sec. IV B.

The Nusselt number scaling for LSV cases at $\mathrm{Pr}=0.1$ [blue symbols at $\mathrm{Ra} / \mathrm{Ra}_{c}<60$ in Fig. 2(b)] is quite steep, $\mathrm{Nu} \sim\left(\mathrm{Ra} / \mathrm{Ra}_{c}\right)^{1.28 \pm 0.02}$. This scaling is in fairly good agreement with the asymptotic scaling $\mathrm{Nu} \sim\left(\mathrm{RaEk}^{4 / 3}\right)^{3 / 2} \sim\left(\mathrm{Ra} / \mathrm{Ra}_{c}\right)^{1.5}$ for the geostrophic turbulence regime reported in Ref. [14] [see dash-dotted line in Fig. 2(b)]. Similar to the $\mathrm{Nu}_{*} \mathrm{vs}^{\mathrm{Ra}} \mathrm{a}_{*}$ scaling in Ref. [43], the asymptotic scaling is, in its full form $\mathrm{Nu} \propto \operatorname{Pr}^{-1 / 2}\left(\operatorname{RaEk}^{4 / 3}\right)^{3 / 2}$, independent of $\kappa$ and $v$. This therefore confirms that in the observed turbulent LSV state, diffusive processes are overwhelmed by nonlinear effects, as expected for highly supercritical flows. We also evaluate the scaling behavior of our $\mathrm{Pr}=0.1$ data in the LSV regime in terms of $\mathrm{Nu}_{*}$ in Fig. 2(c). We find that, for the five cases at $\mathrm{Pr}=0.1$ and with the lowest $\mathrm{Ra} / \mathrm{Ra}_{c}, \mathrm{Nu}_{*}$ scales as $\mathrm{Ra}_{*}^{0.574 \pm 0.002}$, which is in very good agreement with the above-mentioned $\mathrm{Ra}_{*}^{0.55}$ scaling.

Finally, in the rotation-affected regime at $\mathrm{Ra} / \mathrm{Ra}_{c}>60$ (at $\operatorname{Pr}=0.1$ ), $-\partial\langle T\rangle / \partial z$ continues to decrease with $\mathrm{Ra} / \mathrm{Ra}_{c}$, though seemingly more steeply, as the influence of rotation weakens and the bulk becomes more isothermal. This steeper decrease is consistent with that observed beyond RIT in Ref. [13]. In our RA regime, the heat transport scaling is shallower than for LSVs, as it approaches the scaling $\mathrm{Ra}^{1 / 5}$ (i.e., Ek is a constant) for nonrotating Rayleigh-Bénard convection [at $\mathrm{Pr}=0.1$; black short-dashed line in Fig. 2(b)], predicted in Refs. [38,40,41].

\section{B. Boundary layers}

In this section we investigate the vertical profiles of RMS temperature $T_{\mathrm{RMS}}(z)$ and horizontal velocity $u_{\mathrm{RMS}}(z)$ in the region close to the no-slip walls. In Fig. 3 we present these profiles near the bottom wall for selected cases at $\operatorname{Pr} \approx 5$; other cases at different $\mathrm{Ra} / \mathrm{Ra}_{c}$ and $\operatorname{Pr}$ display profiles 
that are qualitatively similar to the ones shown in this figure. In the vertical profile of $T_{\mathrm{RMS}}$, in Fig. 3(a), we see that temperature fluctuations approach zero toward the bottom wall (also toward the top wall, not shown). There the temperature field must meet the constant-temperature boundary condition $T=1$ at $z=0$ (for the top wall $T=0$ at $z=1$ ). Away from the wall, but still at a short distance from it, $T_{\text {RMS }}$ increases rapidly. In fact, $T_{\text {RMS }}$ attains its maximum values in this region. The location of the peak value in the vertical $T_{\mathrm{RMS}}$ profile is often used as a measure of the thickness $\delta_{\theta}$ of the thermal BL. For the case with the lowest supercriticality, at $\mathrm{Ra} / \mathrm{Ra}_{c}=1.3$ [blue curve in Fig. 3(a)], the signature of the thermal layer is much weaker compared to the other cases, which reflects the near-laminar state of the flow at such a low supercriticality.

Vertical profiles of $u_{\text {RMS }}$ are shown in Fig. 3(b) (as solid lines) for the selected cases at $\operatorname{Pr} \approx 5$, and in Fig. 3(c) for two selected cases at $\operatorname{Pr}=0.1$. The profiles at $\mathrm{Ra} / \mathrm{Ra}_{c}=1.3,3.5$, and 7.5 correspond to cases within the cellular, columnar, and plumes regimes, respectively. In these regimes the bulk is expected to be predominantly geostrophic, and as such the near-wall profile of $u_{\text {RMS }}$ should be described by linear Ekman BL theory [16], namely, by the expression $u(z)=u^{\prime}\left[1-2 \cos \left(z / \delta_{u}^{\prime}\right) \exp \left(-z / \delta_{u}^{\prime}\right)+\exp \left(-2 z / \delta_{u}^{\prime}\right)\right]^{1 / 2}$ (see the Appendix), where $u^{\prime}$ and $\delta_{u}^{\prime}$ are parameters describing the (theoretical) geostrophic velocity in the bulk and the BL thickness, respectively. In Figs. 3(b) and 3(c), we confirm this by direct comparison of the numerically determined profiles (solid lines) with their corresponding analytical Ekman BL profiles (dotted lines). Each profile is fitted independently, using $u^{\prime}$ and $\delta_{u}^{\prime}$ as fitting parameters, within the range $0<z<3 \times 10^{-3}$ to fully capture the BL region. Owing to the selected $z$-range, the fitted values of $u^{\prime}$ somewhat reflect the peak value of $u_{\text {RMS }}$ near the wall [see Fig. 9(a) in the Appendix]. Note that the position of the peak value of $u_{\mathrm{RMS}}$, similar to $T_{\mathrm{RMS}}$, is frequently used to define the thickness $\delta_{u}$ of the kinetic BL $[14,15]$; here, we also adopt this definition. The fitted values of $\delta_{u}^{\prime}$ are found to be roughly two times smaller than $\delta_{u}$ for all geostrophic cases [see Fig. 9(b) in the Appendix]. Figures 3(b) and 3(c) thus reveal the excellent agreement between the theoretical profiles and our numerical results close to the walls. Naturally, beyond the BL region, convection in the bulk leads to significant deviations from the theoretical quasisteady behavior, even when the bulk does remain in geostrophic balance at leading order. The fourth case, at $\mathrm{Ra} / \mathrm{Ra}_{c} \approx 80$, displays LSVs and is found within the RIT regime (see discussion in Sec. IV A). Notably, also in this case the near-wall $u_{\mathrm{RMS}}(z)$ profile is consistent with Ekman BL theory, indicating that LSVs in the RIT regime are in fact in leading geostrophic balance. Not surprisingly, also the LSV case at $\operatorname{Pr}=0.1$ and $\mathrm{Ra} / \mathrm{Ra}_{c} \approx 20$ [see Fig. 3(c)], found within the geostrophic turbulence regime, exhibits a $u_{\text {RMS }}$ profile that is well described by Ekman BL theory. In the case at $\mathrm{Ra} / \mathrm{Ra}_{c} \approx 489$, displaying rotation-affected convection, the near-wall region is poorly described by the theoretical profile, which clearly exposes the lack of rotational constraint in this case. Below we further evaluate the rotational constraint of the discussed cases, and of the rest of the cases in our suite of simulations, by considering the kinetic BL thickness, $\delta_{u}$, and evaluating its compliance with the theoretical scaling $\mathrm{Ek}^{1 / 2}$.

Figure 4(a) presents the plots of the thermal and kinetic BL thicknesses, $\delta_{\theta}$ and $\delta_{u}$, as a function of $\mathrm{Ra} / \mathrm{Ra}_{c}$ for all our simulations at all Prandtl numbers. Let us first discuss the kinetic BL thickness. We note that $\delta_{u}$ remains constant for our simulations at $\operatorname{Pr} \approx 5$ and 100 , and fixed Ek: $\delta_{u} \approx 1.7 \times$ $10^{-3}$ for cases at Ek $=3 \times 10^{-7}$ (red and green triangles) and $\delta_{u} \approx 10^{-3}$ for those at Ek $=10^{-7}$ (orange triangles). For cases at $\operatorname{Pr}=0.1$, where instead $\mathrm{Ek}$ is varied and $\mathrm{Ra}$ is kept fixed, the kinetic BL thickness varies with $\mathrm{Ra} / \mathrm{Ra}_{c}$. To evaluate the Ek dependence of the kinetic BL thickness, we plot $\delta_{u} / \mathrm{Ek}^{1 / 2}$ as a function of the supercriticality of the flow in Fig. 4(b). As expected from the previous analysis on the vertical profile of $\delta_{u}$, all cases displaying cells, convective Taylor columns, plumes, and LSVs (in both the regimes of geostrophic turbulence and RIT), at all Prandtl numbers, comply with the $\mathrm{Ek}^{1 / 2}$ scaling with an RMS error just under $4 \%$. Even the prefactor, approximately equal to 3.24 [see linear fit in Fig. 4(b)], is only weakly dependent on Pr. However, in the rotationaffected regime (at $\operatorname{Pr}=0.1$ ), $\delta_{u}$ does not exhibit the theoretical scaling $\mathrm{Ek}^{1 / 2}$. This is consistent with the subdominant role of rotation in this regime. Therefore, the kinetic $\mathrm{BL}$ at $\mathrm{Ra} / \mathrm{Ra}_{c}$ values beyond RIT is certainly not of Ekman type. 

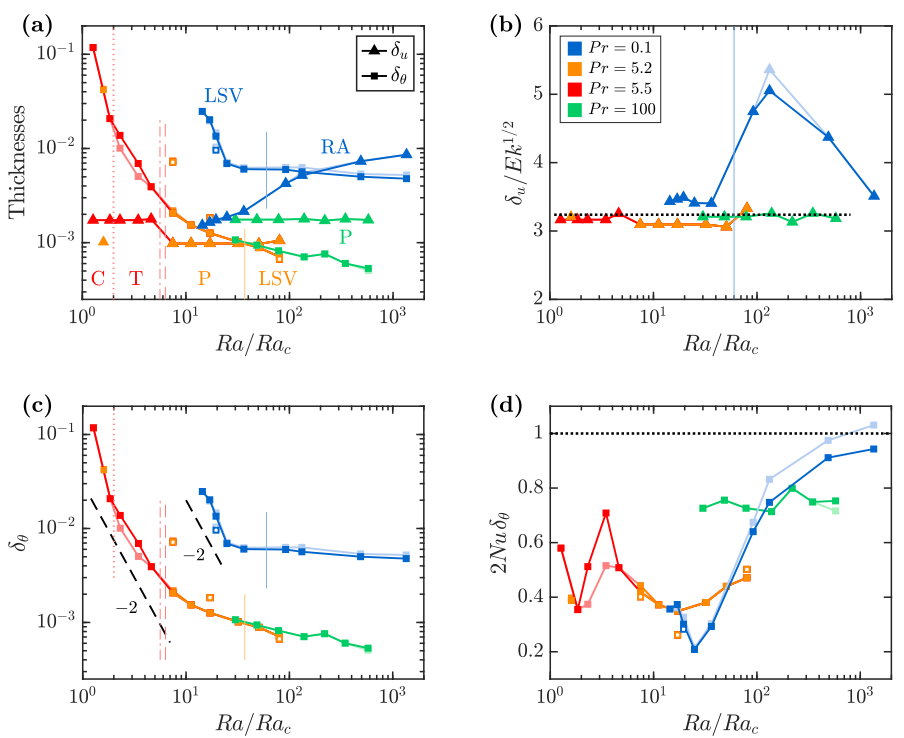

FIG. 4. (a) Kinetic and thermal BL thicknesses, $\delta_{u}$ and $\delta_{\theta}$, near the bottom wall (dark symbols) and the top wall (light symbols), vs $\mathrm{Ra} / \mathrm{Ra}_{c}$. Filled and open symbols are for no-slip and stress-free simulations, respectively. Red, orange, green and blue symbols are cases at $\operatorname{Pr}=5.5\left(\right.$ at $\left.\mathrm{Ek}=3 \times 10^{-7}\right), 5.2\left(\right.$ at Ek $\left.=10^{-7}\right)$, 100 (at Ek $=3 \times 10^{-7}$ ), and 0.1 (at fixed $\mathrm{Ra}=10^{10}$ and varying Ek), respectively. Color-coded vertical lines and regime labels are as in Fig. 2. (b) Kinetic BL thickness scaled by $\mathrm{Ek}^{1 / 2}$, the theoretical scaling for linear Ekman BLs [16]. Most light symbols, corresponding to $\delta_{u}$ near the top wall, lie behind the accompanying dark symbols, corresponding to $\delta_{u}$ near the bottom wall. The horizontal dotted line is at $\delta_{u} / \mathrm{Ek}^{1 / 2} \approx 3.2$. (c) Thermal BL thickness plotted separately for comparison with the asymptotic scaling $\delta_{\theta} \sim\left(\operatorname{RaEk}^{4 / 3}\right)^{-2} \sim\left(\mathrm{Ra} / \mathrm{Ra}_{c}\right)^{-2}$, denoted by dashed lines. (d) Thermal BL thickness multiplied by two times the convective Nusselt number $\mathrm{Nu}$. This quantity is equal to one for nonrotating convection, since there $\delta_{\theta} \approx 1 /(2 \mathrm{Nu})$. In all panels, a case at $\operatorname{Pr}=5.2$ and $\mathrm{Ra} / \mathrm{Ra}_{c}=1.6$ is included to demonstrate the overlap between simulations at $\operatorname{Pr}=5.2$ and 5.5.

For the thermal BL thickness [see Fig. 4(a)], starting from the data at $\operatorname{Pr} \approx 5$, we see that it is thickest at the lowest supercriticality and that it decreases with $\mathrm{Ra} / \mathrm{Ra}_{c}$. This decrease is quite steep for low values of $\mathrm{Ra} / \mathrm{Ra}_{c}$, and gradually becomes shallower toward larger supercriticalities, although no saturation is observed. In Ref. [14] it has been observed that in all geostrophic regimes, over a wide range of Prandtl numbers, $\delta_{\theta}$ roughly scales as $\left(\mathrm{RaEk}^{4 / 3}\right)^{-2}$ in the asymptotic limit of rapid rotation. This scaling is indicated by the black dashed lines (one near the data at $\operatorname{Pr}=0.1$ and another for $\operatorname{Pr} \approx 5$ ) in Fig. 4(c), where we have plotted $\delta_{\theta} \mathrm{vs} R \mathrm{Ra} / \mathrm{Ra}_{c}$ separately for clarity. We note that our simulation data within the cellular and columnar regimes is somewhat consistent with the steep asymptotic scaling. However, deviations from this scaling are already observed toward the plumes regime. This is likely associated with the following: (1) There is enhancement of convective instabilities in the thermal BL, due to Ekman pumping, which leads to thickening of the thermal BL with respect to the trend $\left(\mathrm{RaEk}^{4 / 3}\right)^{-2}$. (2) At finite rotation rate, the increase in thermal forcing renders the thermal plumes more and more confined to the BL to a lesser degree than in the asymptotic case. Remarkably, at $\operatorname{Pr}=0.1, \delta_{\theta}$ agrees very well with the asymptotic scaling, perhaps because the enhanced thermal plumes are shorter lived at this Prandtl number, and fail to prompt the thickening of the thermal BL (as observed for plumes at $\operatorname{Pr} \approx 5$ ). This agreement seems to be best for cases displaying LSVs. At larger $\mathrm{Ra} / \mathrm{Ra}_{c}$, the data show a much shallower scaling. This virtually constant behavior suggests a saturation of $\delta_{\theta}$ toward and within the RA regime, where no Ekman-type BLs develop. In fact, for the cases with largest $\mathrm{Ra} / \mathrm{Ra}_{c}, \delta_{\theta}$ hints a tendency toward behaviors proper of nonrotating convection. Namely, in nonrotating convection, 
$\delta_{\theta}$ relates to the convective Nusselt number $\mathrm{Nu}$ through the expression $\delta_{\theta}=1 /(2 \mathrm{Nu})$ (recall that $\delta_{\theta}$ is already normalized by $H$ ). In Fig. 4(d) we plot the quantity $2 \mathrm{Nu} \delta_{\theta}$ for all simulation cases. We observe that rotationally dominated regimes are poorly described by $2 \mathrm{Nu} \delta_{\theta}=1$, whereas cases at $\operatorname{Pr}=0.1$ in the rotation-affected regime approach this theoretical prediction as $\mathrm{Ra} / \mathrm{Ra}_{c}$ increases. Similar results are reported in Ref. [49] for the rotationally constrained regime at $\mathrm{RaEk}^{3 / 2}<10$, or $\mathrm{Ra} / \mathrm{Ra}_{c} \lesssim 124\left(\right.$ at $\mathrm{Pr}=0.1$ and $\mathrm{Ra}=10^{10}$ ) and weakly rotating convection at $\mathrm{RaEk}^{3 / 2}>10$, or $\mathrm{Ra} / \mathrm{Ra}_{c} \gtrsim 124$.

Finally, we note the following. For cells and columns, the thermal BL is thicker than the kinetic BL. This is also observed in the plumes regime, although, in contrast, plumes at $\operatorname{Pr}=100$ exhibit a thicker kinetic BL. A similar situation occurs in the LSV regime: $\delta_{u}>\delta_{\theta}$ for LSVs at $\operatorname{Pr} \approx 5$, whereas $\delta_{\theta}>\delta_{u}$ for those at $\operatorname{Pr}=0.1$. This all suggests that the bulk flow state does not significantly depend on the relative thickness of the boundary layers. Nonetheless, the arrangement of the boundary layers influences the distribution of flow- and temperature structures near the walls. This is the subject of study of the subsequent sections.

\section{Skewness of temperature, velocity, and vorticity}

To gain insight into the temperature and velocity structures in the flow, we draw upon the characterization of the probability distribution of related physical quantities. In particular, in this section we evaluate the third-order moment, or skewness, of these distributions. In Sec. I we introduced that the skewness of a probability distribution provides a measure of its asymmetry. Hence, positive values of the temperature skewness $T_{\text {SKEW }}=\left\langle(T-\langle T\rangle)^{3}\right\rangle / T_{\text {RMS }}^{3}$ indicate that large above-average temperature fluctuations are localized in small portions of the domain, whereas smaller below-average values are distributed over a larger portion of the domain; and vice versa for a negatively skewed distribution. This is also valid for the skewness of vertical velocity $w_{\text {SKEW }}=\left\langle w^{3}\right\rangle / w_{\text {RMS }}^{3}$ and of vertical vorticity $\omega_{z \text {, SKEW }}=\left\langle\omega_{z}^{3}\right\rangle / \omega_{z}^{3}$, RMS . As before, $\langle\cdot\rangle$ denotes averaging over time and over horizontal planes at a given height; thus, both $\langle w\rangle$ and $\left\langle\omega_{z}\right\rangle$ are zero at all heights. Figure 5 shows the vertical profiles of skewness for selected cases at $\operatorname{Pr} \approx 5$. For most cases, including those at different $\mathrm{Ra} / \mathrm{Ra}_{c}$ and Pr not shown in Figs. 5(a) to 5(c), the vertical profiles of $T_{\text {SKEW }}$ and $w_{\text {SKEW }}$ are antisymmetric about midheight, whereas the profiles of $\omega_{z}$, SKEW are symmetric. This is certainly expected, as it is consistent, for instance, with the presence of localized hot rising cyclonic flows in the lower half of the domain and localized cold sinking cyclonic flows in the upper half $[15,19,21,50,51]$. However, we notice that the vertical profiles of $T_{\text {SKEW }}$ and $w_{\text {SKEW }}$ at $\operatorname{Pr} \approx 5$ and $\mathrm{Ra} / \mathrm{Ra}_{c}=3.5$ are clearly not antisymmetric, and the profile of $\omega_{z}$, SKEW is not symmetric. The predominantly positive profiles of $T_{\text {SKEW }}$ and $w_{\text {SKEW }}$ indicate that the flow is dominated by localized hot rapidly rising convective columns surrounded by cool slowly sinking fluid. This is, e.g., reflected in Fig. 1(b), where the number of hot columns is larger than the number of cold columns. A similar situation is found for the case at $\operatorname{Pr} \approx 5$ and $R a / R_{c}=2.3$ (not shown). A possible reason for this asymmetry may be that this arrangement has emerged from the randomly perturbed initial condition during the early development of the columnar structures, and it then persists over time due to the strong stability of the flow at low supercriticality. The net effect is that skewness curves in Figs. 5(a) and 5(b) are shifted toward positive values, while they should be zero at $z=0.5$, and $\omega_{\text {SKEW }}$ should be vertically symmetric [Fig. 5(c)]. We expect that this asymmetric arrangement will equilibrate into the expected distribution with equal number of hot rising and cold sinking columns at sufficiently long simulation times, of the order of the viscous diffusion timescale $\tau_{v}=\tau_{\text {ff }} \sqrt{\mathrm{Ra} / \operatorname{Pr}}$ or $\sim 10^{4}$ convective time units (at $\mathrm{Ra} \sim 10^{10}$ and $\operatorname{Pr} \approx 5$ ). We must acknowledge, however, that the question why this is not observed in other cases in the columnar regime, or even for cells and plumes, warrants further investigation.

For most cases, the largest values of $T_{\text {SKEW }}, w_{\text {SKEW }}$ and $\omega_{z \text {, SKEW }}$ are found near the walls. This reveals a strong asymmetry in the statistical distribution of temperature, vertical velocity, and vertical vorticity in this region. In Figs. 5(d) and 5(f) we plot the values of skewness at the bottom kinetic boundary layer $\left(z=\delta_{u}\right.$; dark symbols) and at the top kinetic boundary layer $\left(z=1-\delta_{u}\right.$; 
(a)

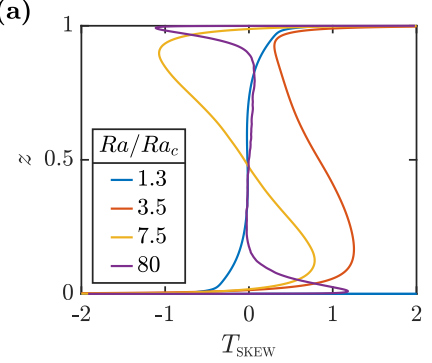

(d)

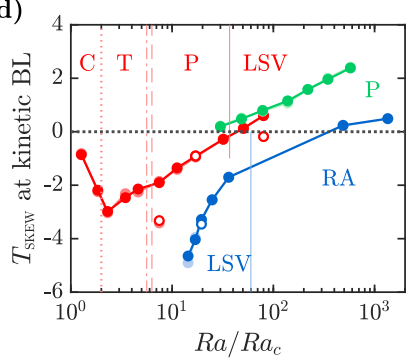

(b)

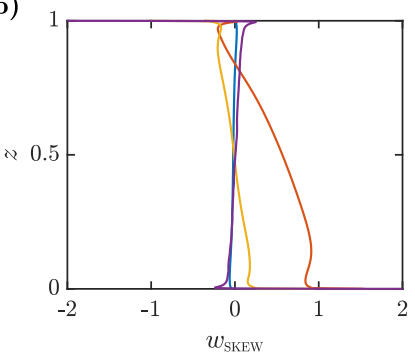

(e)

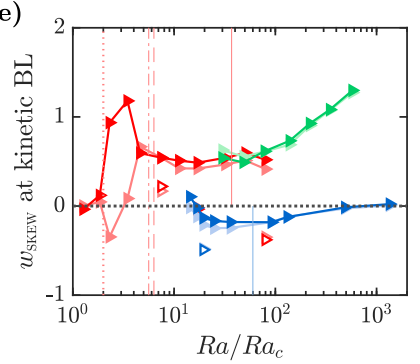

(c)

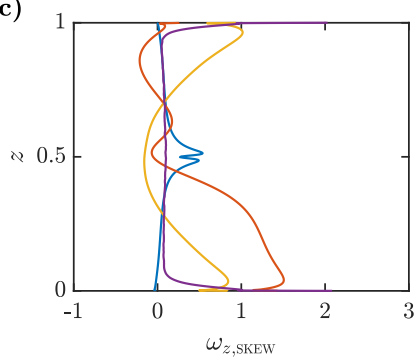

(f)

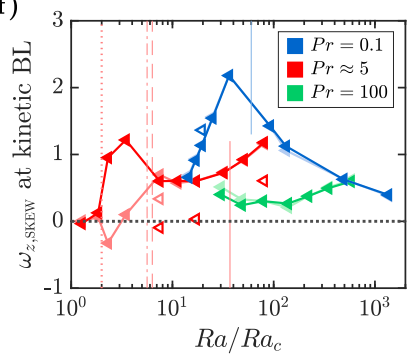

FIG. 5. Vertical profiles of skewness of (a) temperature, (b) vertical velocity, and (c) vertical vorticity, for selected cases at $\operatorname{Pr} \approx 5$. Skewness of (d) temperature, (e) vertical velocity, and (f) vertical vorticity at both the bottom kinetic BL $\left(z=\delta_{u}\right.$; dark symbols) and the top kinetic BL $\left(z=1-\delta_{u}\right.$; light symbols; $T_{\text {SKEW }}$ and $w_{\text {SKEW }}$ are multiplied by minus one). Filled and open symbols are for no-slip and stress-free simulations, respectively. Color-coded vertical lines and regime labels are as in Fig. 2.

light symbols), as a function of $\mathrm{Ra} / \mathrm{Ra}_{c}$. The values of $T_{\text {SKEW }}$ and $w_{\text {SKEW }}$ at $z=1-\delta_{u}$ are plotted with a minus sign for comparison of their magnitude with that at $z=\delta_{u}$. Notice the excellent agreement of all skewness values at these two heights for most of our simulation cases, barring the aforementioned cases at $\mathrm{Ra} / \mathrm{Ra}_{c}=2.3$ and 3.5, both at $\operatorname{Pr} \approx 5$, where the values of $w_{\text {SKEW }}$ and $\omega_{z}$, SKEW are noticeably different. This discrepancy results from the above-mentioned asymmetry in these two cases, yet remarkably $T_{\mathrm{SKEW}}\left(z=\delta_{u}\right) \approx-T_{\mathrm{SKEW}}\left(z=1-\delta_{u}\right)$. Bearing in mind these expected symmetries and antisymmetries, let us then use measurements taken at the bottom kinetic BL as a starting point for our discussions.

In Figs. 5(e) and 5(f), we see that, for all cases at $\operatorname{Pr} \approx 5, w_{\text {SKEW }}$ and $\omega_{z \text {, SKEW }}$ are positive, which reveals the presence of localized flows with cyclonic vorticity emanating from the BL [see flow visualizations in Figs. 6(b) and 6(c)]. However, $T_{\text {SKEW }}<0$ for the cases displaying cells, columns and plumes. Interestingly, in Sec. IV B we observed that in these cases the kinetic BL is embedded within the thermal BL [i.e., $\delta_{u}<\delta_{\theta}$ in Fig. 4(a)]. This indicates that the localization of cyclonic rising flows within the thermal boundary layer is not matched by localization of hot fluid in this region. Instead, the hot fluid in the thermal BL interior is distributed over large portions of the horizontal domain [large red patches in Fig. 6(a)], whereas the cold fluid parcels that penetrate into the thermal BL are concentrated in smaller regions [the area occupied by the blue patches in Fig. 6(a) is about 1.7 times smaller than for red patches]. In contrast, for LSV cases (at $\operatorname{Pr} \approx 5$ ), where the kinetic BL becomes thicker than the thermal BL, $T_{\text {SKEW }}$ becomes positive, indicating that hot fluid parcels become localized [e.g., in Fig. 6(d), red patches occupy $45 \%$ of the total cross-sectional area]. Therefore, in these cases, localization of cyclonic rising flows at $z=\delta_{u}\left(>\delta_{\theta}\right)$ is matched by concentrated regions of hot fluid at this height [Figs. 6(e) and 6(f)]. Other cases for which $w_{\text {SKEW }}>0$ and/or $\omega_{z \text {, SKEW }}>0$ is matched by $T_{\text {SKEW }}>0$ are those displaying plumes at $\operatorname{Pr}=100$, and the two cases at $\mathrm{Pr}=0.1$ with largest $\mathrm{Ra} / \mathrm{Ra}_{c}$; all these cases are also characterized by $\delta_{u}>\delta_{\theta}$. For the remaining cases where $\delta_{u}<\delta_{\theta}$, i.e., the five cases at $\operatorname{Pr}=0.1$ with lowest $\mathrm{Ra} / \mathrm{Ra}_{c}$, the statistical distribution of temperature is negatively skewed $\left(T_{\text {SKEW }}<0\right)$, even when 

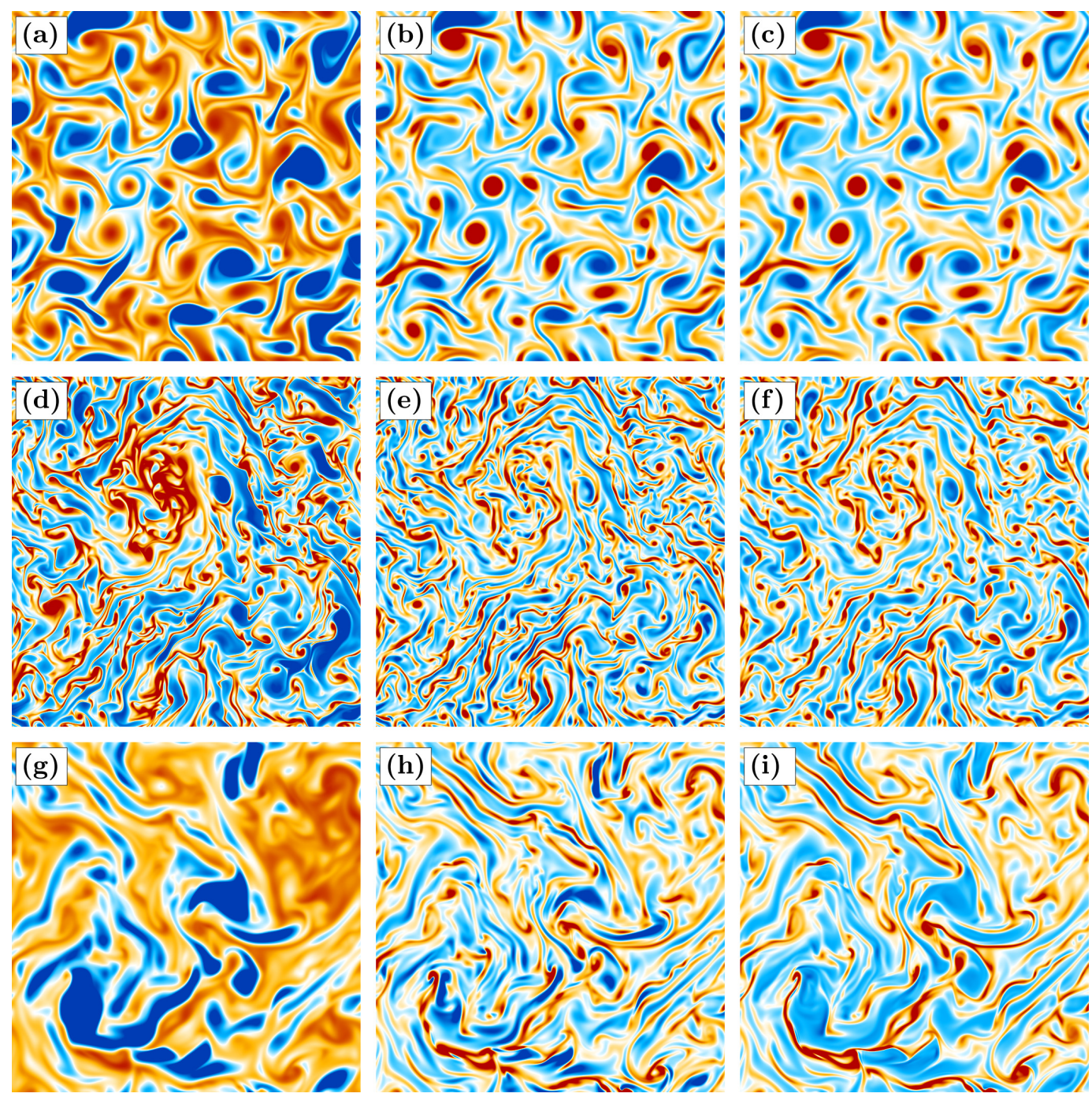

FIG. 6. Snapshots of temperature fluctuations (left), vertical velocity (center), and vertical vorticity (right), at the kinetic $\mathrm{BL}$ edge $\left(z=\delta_{u}\right)$ for cases at $\operatorname{Pr} \approx 5$ and $\mathrm{Ra} / \mathrm{Ra} \mathrm{a}_{c}=7.5$ (top), at $\operatorname{Pr} \approx 5$ and $\mathrm{Ra} / \mathrm{Ra} \mathrm{a}_{c}=80$ (middle), and at $\mathrm{Pr}=0.1$ and $\mathrm{Ra} / \mathrm{Ra}_{c}=20$ (bottom). In panels (b) and (c), vertical-velocity and verticalvorticity features, respectively, bear a strong resemblance between them.

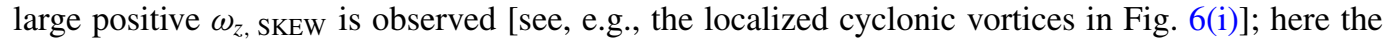
vertical-velocity skewness is very small. Let us summarize the findings discussed above. For clarity, we shall consider only the skewness statistics near the bottom wall, while keeping in mind that the same conclusions can be drawn for the statistics near the top wall for temperature features with opposite thermal contrast. When $\delta_{u}>\delta_{\theta}$, localized cyclonic flows from the kinetic BL concur with concentrated parcels of hot fluid at $z=\delta_{u}$, while nonlocalized anticyclonic flows into the BL are matched by widespread regions of cold fluid. By zooming in on the near-wall region in Fig. 5(a) for all our simulation cases (not shown), we note that, intuitively, the aforementioned results on the spatial distribution of temperature are roughly consistent with a negatively skewed temperature distribution within the thermal $\mathrm{BL}$, and a positively skewed temperature distribution in the region outside the thermal BL. When $\delta_{u}<\delta_{\theta}$, localized cyclonic motion of fluid emanating from the kinetic 
$\mathrm{BL}$ is correlated with widespread regions of hot fluid within the thermal BL, whereas large regions of weak anticyclonic flow into the BL are correlated with small regions of cold fluid. Here, similar to the cases where $\delta_{u}>\delta_{\theta}$, the temperature distribution is negatively skewed within the thermal BL, nonetheless this distribution is not necessarily positively skewed in the region outside the thermal BL [i.e., even in the region outside this BL, hot plumes may continue to occupy a larger portion of the horizontal domain compared to cold plumes]. This indeed suggests that the thermal BL does not necessarily nucleate concentrated parcels of hot fluid that match the localized cyclonic flows from the kinetic BL.

The relative thickness of the boundary layers appears to determine the statistical distribution of temperature at the kinetic BL, although, as concluded in Sec. IV B, the BL arrangement does not further influence the bulk flow state. Hence, by extension, the resulting asymmetries in the near-wall distribution of temperature may not influence the bulk either. In the next section, we investigate flow and temperature structures, both in the bulk and near the walls, based on the feasibility of extreme values in the statistical distributions discussed in this section.

\section{Kurtosis of temperature, velocity, and vorticity}

Further understanding of the flow and temperature structures can be gained by computing the kurtosis of the probability distribution of relevant physical quantities. The kurtosis measures the feasibility of extreme values in the distribution, e.g., large values of kurtosis indicate that extreme fluctuations are more likely to occur. For reference, in homogeneous isotropic turbulence (HIT) the velocity distribution is practically Gaussian, with kurtosis equal to 3 [52,53], whereas the vorticity distribution is strongly non-Gaussian, with kurtosis larger than 3 and increasing with the turbulence intensity [54,55]. The presence of localized coherent structures in the flow, such as plumes, raises the probability of extreme events, so that kurtosis larger than 3 is expected. We compute the kurtosis of the temperature distribution as $T_{\mathrm{KURT}}=\left\langle(T-\langle T\rangle)^{4}\right\rangle / T_{\mathrm{RMS}}^{4}$, of vertical velocity as $w_{\mathrm{KURT}}=\left\langle w^{4}\right\rangle / w_{\mathrm{RMS}}^{4}$, and of vertical vorticity as $\omega_{z, \mathrm{KURT}}=\left\langle\omega_{z}^{4}\right\rangle / \omega_{z, \mathrm{RMS}}^{4}$; recall that $\langle w\rangle,\left\langle\omega_{z}\right\rangle \approx 0$ averaged over time and over horizontal planes at all heights. We present the kurtosis profiles in Fig. 7 for selected cases at $\operatorname{Pr} \approx 5$. Similar to skewness, the kurtosis profiles of the case at $\mathrm{Ra} / \mathrm{Ra}_{c}=3.5$ (also at $\mathrm{Ra} / \mathrm{Ra}_{c}=2.3$, not shown) display asymmetries about midheight, albeit less evident than for skewness due to the even parity of the kurtosis function. Figures 7(a) and 7(c) show that the likelihood of extreme fluctuations in the bulk varies with supercriticality. In Figs. 7(d) to 7(f), we plot the kurtosis values at midheight as a function of $\mathrm{Ra} / \mathrm{Ra}_{c}$ for all cases.

At $\operatorname{Pr} \approx 5$ (red symbols), all values of kurtosis ( $T_{\mathrm{KURT}}, w_{\mathrm{KURT}}$ and $\left.\omega_{z, \mathrm{KURT}}\right)$ are close to 3 at our lowest supercriticality (in the cellular regime), they then increase with $\mathrm{Ra} / \mathrm{Ra}_{c}$ throughout the cellular and columnar regimes, they decrease in the plumes regime (also observed for plumes at $\operatorname{Pr}=100$; see green symbols), and finally $T_{\text {KURT }}$ and $w_{\text {KURT }}$ are again close to 3 in the LSV regime (also observed for LSVs at $\operatorname{Pr}=0.1$; see blue symbols; recall, for reference, that the velocity distribution in HIT is approximately Gaussian, with kurtosis equal to 3). These changes of $w_{\text {KURT }}$ with supercriticality are also reported in Ref. [14] at various Prandtl numbers. Notably, for LSV cases, the vertical-vorticity kurtosis remains larger than $3: \omega_{z, \text { KURT }} \approx 4$ and potentially increasing at $\operatorname{Pr} \approx 5$, and increasing $\omega_{z, \text { KURT }}>5$ at $\operatorname{Pr}=0.1$. This indicates that, for LSV cases, the likelihood of large values of vertical vorticity increases with supercriticality (in HIT, the vorticity kurtosis is larger than 3 and increasing with the turbulence intensity). Notice that the above-discussed kurtosis values do not appear to discern between the regimes of geostrophic turbulence and rotation-influenced turbulence (RIT). Namely, the underlying turbulent state in these two regimes yields a similar trend for $T_{\text {KURT }}, w_{\text {KURT }}$, and $\omega_{z}$, KURT with varying flow supercriticality. Finally, in the rotation-affected regime at $\operatorname{Pr}=0.1$, the turbulent bulk yields a Gaussian distribution for temperature and vertical velocity $\left(T_{\mathrm{KURT}}, w_{\mathrm{KURT}} \approx 3\right)$, whereas the vertical-vorticity kurtosis is $\omega_{z \text {, KURT }} \approx 10$. This value of $\omega_{z}$, KURT is larger than for LSV cases, indicating that extreme values of $\omega_{z}$ are more likely to occur in the rotation-affected regime. Furthermore, $\omega_{z}$, KURT remains nearly constant for all cases in this regime. 
(a)
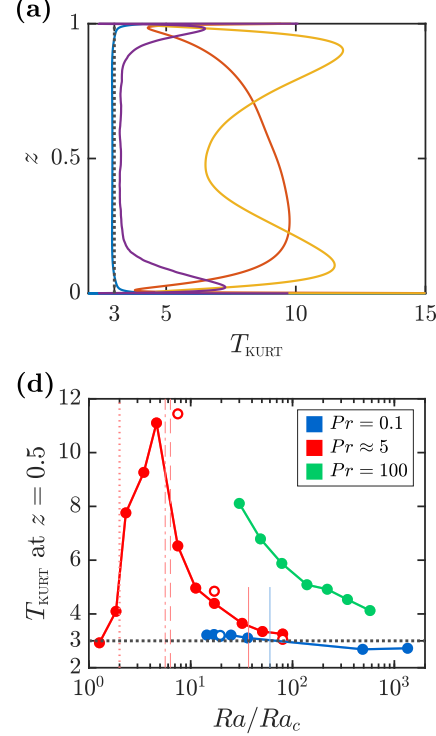

(b)

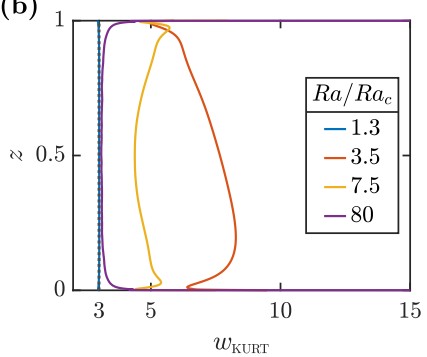

(e)

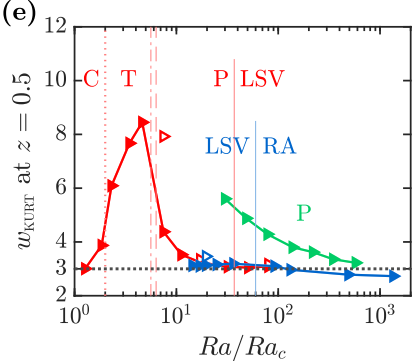

(c)

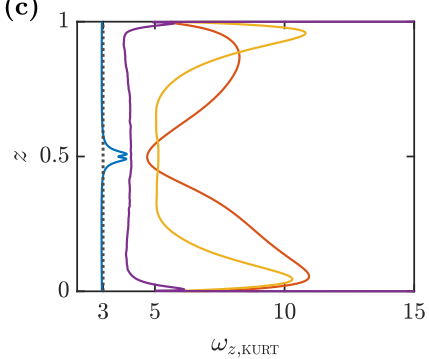

(f)

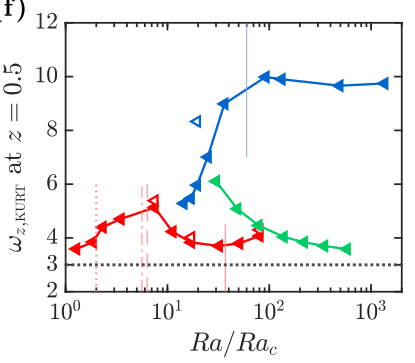

FIG. 7. Vertical profiles of kurtosis of (a) temperature, (b) vertical velocity, and (c) vertical vorticity, for selected cases at $\operatorname{Pr} \approx 5$. Kurtosis of (d) temperature, (e) vertical velocity, and (f) vertical vorticity at midheight $(z=0.5)$ plotted against $\mathrm{Ra} / \mathrm{Ra}_{c}$ for all simulation cases. Filled and open symbols are for no-slip and stress-free simulations, respectively. Color-coded vertical lines and regime labels are as in Fig. 2.

In Fig. 8 we plot the kurtosis values, both at the bottom kinetic boundary layer (dark symbols) and at the top kinetic boundary layer (light symbols), as a function of flow supercriticality. At $\operatorname{Pr} \approx 5$, similar to the bulk, $T_{\text {KURT }}, w_{\text {KURT }}$, and $\omega_{z \text {, KURT }}$ increase with $\mathrm{Ra} / \mathrm{Ra}_{c}$ in the cellular and columnar regimes, and then decrease in the plumes regime. This indicates that, also in the near-wall region, the likelihood of extreme values of temperature, vertical velocity, and vertical vorticity increases with supercriticality within the cellular and columnar regimes, and decreases in the plumes regime. For plumes at $\operatorname{Pr}=100$, the behavior of $T_{\mathrm{KURT}}, w_{\mathrm{KURT}}$, and $\omega_{z \text {, KURT }}$ is markedly different (also different from its behavior in the bulk). We observe that $T_{\mathrm{KURT}}$ and $w_{\mathrm{KURT}}$ increase with $\mathrm{Ra} / \mathrm{Ra} \mathrm{c}_{c}$ instead, whereas $\omega_{z, \text { KURT }} \approx 5$ remains approximately unchanged for the parameter values considered. This implies that, for plumes at this Prandtl number, there is an increasing probability of localized hot rising (cold sinking) fluid near the bottom (top) wall [consistent with positive (negative) values

(a)

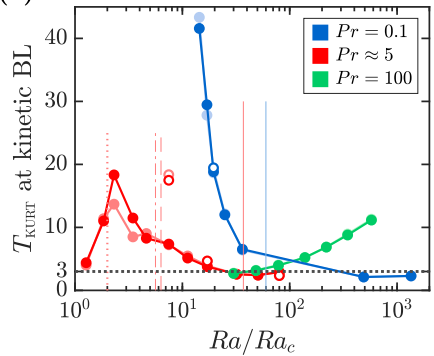

(b)

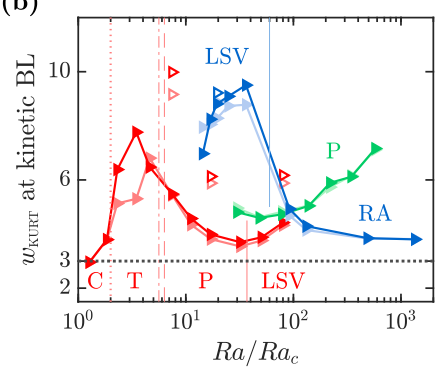

(c)

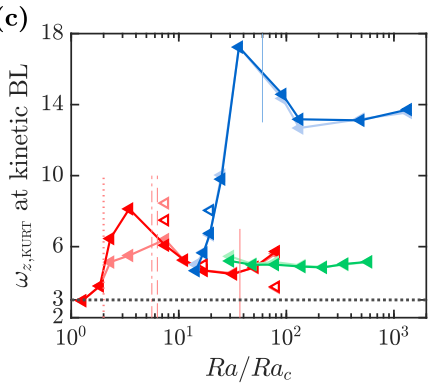

FIG. 8. (a) Temperature, (b) vertical-velocity, and (c) vertical-vorticity kurtosis at the bottom kinetic BL $\left(z=\delta_{u}\right.$; dark symbols) and at the top kinetic $\mathrm{BL}\left(z=1-\delta_{u}\right.$; light symbols) vs $\mathrm{Ra} / \mathrm{Ra}_{c}$ for all simulations cases. Filled and open symbols are for no-slip and stress-free simulations, respectively. Color-coded vertical lines and regime labels are as in Fig. 2. 


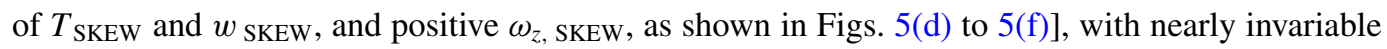
likelihood of large values of cyclonic vorticity. This can be understood as the prevalence of coherent, longer-lived flow structures near the walls owing to the larger Prandtl number.

For LSV cases at $\operatorname{Pr} \approx 5$, and also at $\operatorname{Pr}=0.1$, the vertical-velocity kurtosis presents considerable differences in comparison to the bulk: it is larger than 3 and increases with $\mathrm{Ra} / \mathrm{Ra}_{c}$. This indicates an increasing likelihood of large vertical-velocity and vertical-vorticity fluctuations at the Ekman BL. The presence of these near-wall vertical flows may influence the flow far from the walls. The competition between Ekman BL flows and large-scale vortices in the bulk is discussed in Ref. [18]. For LSVs at $\operatorname{Pr}=0.1$, the large values of $T_{\text {KURT }}$ are related to the strong asymmetry in the distribution of temperature in this region [note the large negative values of $T_{\text {SKEW }}$ in Fig. 5(d)]. Namely, strongly localized parcels of cold fluid (large $T_{\text {SKEW }}<0$ ) must necessarily register extreme values of temperature that are well below the mean temperature (large $T_{\mathrm{KURT}}$ ), whereas extended regions of hot fluid register temperatures much closer to the mean. For instance, in the snapshot of temperature fluctuations shown in Fig. 6(g), parcels of fluid with below-average temperature (in blue) occupy $32 \%$ of the total cross-sectional area, and register a mean temperature difference (with respect to the average temperature of the entire cross section) that, in absolute terms, is about two times larger than that for hot parcels (in red). For LSV cases at $\operatorname{Pr} \approx 5$, on the other hand, the asymmetry of the temperature distribution is small, but seemingly increasing [see Fig. 5(d)]. We thus expect that, also at this Prandtl number, $T_{\text {KURT }}$ will eventually become large with increasing $\mathrm{Ra} / \mathrm{Ra}_{c}$ for LSV cases. Finally, in the rotation-affected regime (at $\mathrm{Pr}=0.1$ ), temperature, vertical velocity, and vertical vorticity approach their corresponding statistical distribution for the bulk. That is, Gaussian for temperature and vertical velocity $\left(T_{\mathrm{KURT}}, w_{\mathrm{KURT}} \rightarrow 3\right)$ and larger-than-Gaussian $\left(\omega_{z, \text { KURT }}>3\right)$, but approximately constant, for vertical vorticity.

As a final note, also here it is worthwhile to mention that even when the near-wall values of $T_{\mathrm{KURT}}, w_{\mathrm{KURT}}$ and $\omega_{z}$, KURT, and their variation with $\mathrm{Ra} / \mathrm{Ra}_{c}$, possess diagnostic properties of the flow in this region, these quantities do not seem to distinguish between the LSVs in the geostrophic turbulence regime and those in the RIT regime.

\section{CONCLUSIONS}

We have investigated flow and temperature features of rotating Rayleigh-Bénard convection, both in the bulk and close to no-slip walls, from a statistical perspective. We have employed both flowand temperature-based statistical quantities for distinction and characterization of RRBC regimes, and we have compared our results (at a finite rotation rate) with observations from asymptotic studies (in the asymptotic limit of rapid rotation) [14]. We find that, in the cellular and columnar regimes $($ at $\operatorname{Pr} \approx 5)$, the mean temperature gradient at midheight scales as $\left(R a / R_{c}\right)^{-0.91 \pm 0.07}$, in excellent agreement with results from asymptotic models. Our data for the convective Nusselt number are consistent with the scaling exponent $\gamma=3$ obtained from asymptotic models with parametrized Ekman pumping, and DNS with no-slip walls [37]. For LSV cases (at $\mathrm{Pr}=0.1$ and $\approx 5$ ), our data of the midheight mean temperature gradient resolve two distinct behaviors: one with a saturated $-\left.\partial_{z}\langle T\rangle\right|_{z=0.5} \sim\left(\mathrm{Ra} / \mathrm{Ra}_{c}\right)^{0}$, and another with a decreasing $-\left.\partial_{z}\langle T\rangle\right|_{z=0.5}$ that is well described by $\left(\mathrm{Ra} / \mathrm{Ra}_{c}\right)^{-0.21}$. The former is consistent with the so-called geostrophic turbulence regime [14], and the latter is compatible with the recently experimentally observed regime of so-called rotation-influenced turbulence (RIT) [13]. These results demonstrate the presence of LSVs beyond the geostrophic turbulence regime, and reveal their existence within the RIT regime. The heat transport by LSVs (at $\mathrm{Pr}=0.1$ ) in the geostrophic turbulence regime is in accordance with the diffusion-free asymptotic scaling $\mathrm{Nu} \sim\left(\mathrm{Ra} / \mathrm{Ra}_{c}\right)^{3 / 2}$.

The theoretical scaling from linear Ekman BL theory has been employed as an indicator of rotational control in the fluid layer. For the geostrophic regimes of cells, columns, plumes and geostrophic turbulence, the kinetic BL thickness scales as $\mathrm{Ek}^{1 / 2}$, as predicted in Ekman BL theory [16], a clear indication (and confirmation) that this boundary layer is of Ekman type. The kinetic $\mathrm{BL}$ thickness of LSVs in the RIT regime also complies with the scaling $\mathrm{Ek}^{1 / 2}$, which reveals that, 
also in this regime, the bulk flow is in leading geostrophic balance. For rotation-affected convection, the kinetic BL thickness deviates significantly from the aforementioned scaling, consistent with the loss of rotational constraint in this region. The scaling of the thermal BL thickness in strongly rotationally constrained regimes (cells and columns at $\operatorname{Pr} \approx 5$ ) is roughly described by the asymptotic scaling $\left(\mathrm{Ra} / \mathrm{Ra}_{c}\right)^{-2}$. Toward the plumes regime, and even up to the LSV regime, deviations from this scaling may be observed. In the rotation-affected regime, the thermal BL is marginally affected by rotation, and its thickness may solely depend on the Rayleigh number, as $\delta_{\theta}$ is seen to approach the nonrotating scaling $1 /(2 \mathrm{Nu})$. Hence, at even larger supercriticality, we reiterate the hypothesis in Ref. [13], whereby nonrotating-style thermal boundary layers form.

We have examined flow- and temperature-based statistics in the region near the no-slip walls. Our results suggest that the relative thickness of the kinetic and thermal BLs influences the skewness of the statistical distribution of temperature close to the walls. Remarkably, the observed plumes and LSV flow states develop regardless of the relative thickness of the BLs and therefore, by extension, notwithstanding the near-wall distribution of temperature either. This suggests that these bulk flow states are not dictated by the BL arrangement and the resulting asymmetries in the temperature distribution.

Finally, the kurtosis of temperature, vertical velocity, and vertical vorticity exhibits transitional behaviors at similar $\mathrm{Ra} / \mathrm{Ra}_{c}$ in the bulk and near the walls. These kurtosis values, and their variation with flow supercriticality, closely characterize the flow state, both in the bulk and the BLs, yet they do not decisively discerns between the LSVs in the geostrophic turbulence regime and those in the RIT regime. Indeed, considering the employed flow- and temperature-based statistics, and the explored parameter values, the mean temperature gradient at midheight provides the clearest diagnostic distinguishing between these two regimes. This is certainly of great convenience, as measurements of this quantity can be readily available in laboratory experiments of rapidly rotating turbulent convection.

The study of rotating turbulent convection at extreme parameter values presents challenges in both laboratory experiments and numerical simulations. In the latter approach these challenges are exacerbated by the presence of no-slip walls, as the development of thin viscous boundary layers adjacent to the wall surface demands increased numerical resolution in this region. Hence, much of our knowledge of these systems has been gained from simulations employing stress-free boundary conditions, in particular from those in the asymptotic limit of rapid rotation. Here we provide results from direct numerical simulations at rather extreme parameter values bounded by experimentally realizable no-slip boundary conditions. The provided statistical characterization of RRBC regimes can be used as a framework for identification of these flow states in future experiments and simulations at even more extreme parameter values, and for extrapolation to geophysical and astrophysical flow systems.

\section{ACKNOWLEDGMENTS}

A.J.A.G., M.M., J.S.C., and R.P.J.K. received funding from the European Research Council (ERC) under the European Union's Horizon 2020 research and innovation programme (Grant agreement No. 678634). We are grateful for the support of the Netherlands Organisation for Scientific Research (NWO) for the use of supercomputer facilities (Cartesius) under Grants No. 15462, 16467, 2019.005, and 2020.009.

\section{APPENDIX: THEORETICAL EKMAN BL VERTICAL PROFILE OF HORIZONTAL VELOCITY}

An analytical expression for the near-wall vertical profile of horizontal velocity can be attained from linear Ekman BL theory [16]. Let us assume $\boldsymbol{u}=\left(u_{x}, u_{y}\right)$ the horizontal fluid velocity and 

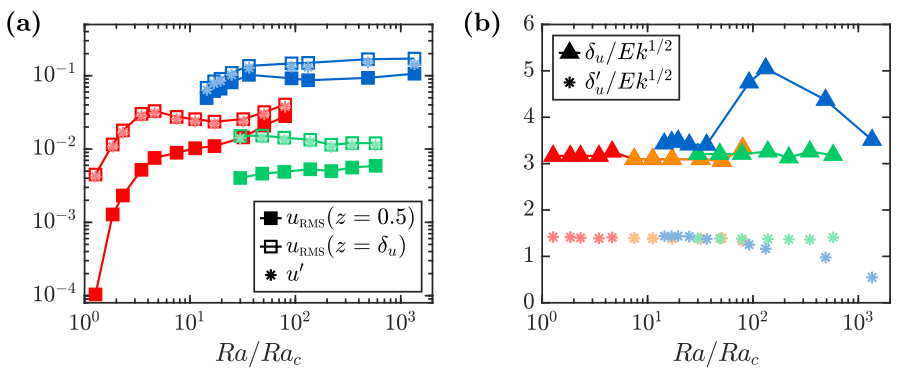

FIG. 9. Fitting parameters $u^{\prime}$ and $\delta_{u}^{\prime}$ for the near-wall vertical profile of $u_{\text {RMS }}$ of all simulation cases at $\operatorname{Pr}=0.1$ (blue), $\operatorname{Pr} \approx 5$ [red in panel (a); and red and orange in panel (b) for consistency with Fig. 4(b)], and $\operatorname{Pr}=100$ (green). In panel (a), we also plot the RMS horizontal velocity $u_{\text {RMS }}$ at midheight $z=0.5$ (filled symbols) and at $z=\delta_{u}$ (open symbols). In panel (b), we include $\delta_{u} / \mathrm{Ek}^{1 / 2}$ (triangles), where $\delta_{u}$ is the location of the peak value of $u_{\text {RMS }}$ close to the bottom wall.

$\boldsymbol{u}^{\prime}=\left(u_{x}^{\prime}, u_{y}^{\prime}\right)$ the theoretical geostrophic velocity in the bulk, so that the velocity components $u_{x}$ and $u_{y}$ are given by

$$
\begin{aligned}
& u_{x}(z)=u_{x}^{\prime}-\left[u_{x}^{\prime} \cos \left(z / \delta_{u}^{\prime}\right)+u_{y}^{\prime} \sin \left(z / \delta_{u}^{\prime}\right)\right] \exp \left(-z / \delta_{u}^{\prime}\right), \\
& u_{y}(z)=u_{y}^{\prime}+\left[u_{x}^{\prime} \sin \left(z / \delta_{u}^{\prime}\right)-u_{y}^{\prime} \cos \left(z / \delta_{u}^{\prime}\right)\right] \exp \left(-z / \delta_{u}^{\prime}\right),
\end{aligned}
$$

where $\delta_{u}^{\prime}$ characterizes the BL thickness. Equations (A1) and (A2) satisfy the boundary conditions: $u_{x}=u_{y}=0$ at $z=0$, and $\left(u_{x}, u_{y}\right) \rightarrow\left(u_{x}^{\prime}, u_{y}^{\prime}\right)$ as $z \rightarrow \infty$. These equations are general analytical solutions to the set of differential equations that results when considering a geostrophic bulk near a no-slip (bottom) boundary; their detailed derivation is presented in Ref. [30] (see Sec. 2.7 therein). By combining Eqs. (A1) and (A2), according to $u=\left(u_{x}^{2}+u_{y}^{2}\right)^{1 / 2}$, we obtain the desired analytical expression for the vertical profile of horizontal velocity:

$$
u(z)=u^{\prime}\left[1-2 \cos \left(z / \delta_{u}^{\prime}\right) \exp \left(-z / \delta_{u}^{\prime}\right)+\exp \left(-2 z / \delta_{u}^{\prime}\right)\right]^{1 / 2},
$$

with $u^{\prime}=\left(u_{x}^{\prime 2}+u_{y}^{\prime 2}\right)^{1 / 2}$. Equation (A3) is employed to fit the numerically determined vertical profile of $u_{\text {RMS }}$ of each of our simulation cases [see, e.g., Figs. 3(b) and 3(c)]. Each profile is fitted independently, using $u^{\prime}$ and $\delta_{u}^{\prime}$ as fitting parameters, within the range $0<z<3 \times 10^{-3}$ [except at $\left(\mathrm{Pr}, \mathrm{Ra} / \mathrm{Ra}_{c}\right)=(0.1,489)$ where the range $0<z<5 \times 10^{-2}$ is used], to fully capture the near-wall region. In Fig. 9 we plot the fitted values of $u^{\prime}$ and $\delta_{u}^{\prime}$ (the latter normalized by $\mathrm{Ek}^{1 / 2}$ ) for each simulation case.

[1] N. Schaeffer, D. Jault, H.-C. Nataf, and A. Fournier, Turbulent geodynamo simulations: A leap towards Earth's core, Geophys. J. Int. 211, 1 (2017).

[2] J. Aubert, T. Gastine, and A. Fournier, Spherical convective dynamos in the rapidly rotating asymptotic regime, J. Fluid Mech. 813, 558 (2017).

[3] C. Guervilly, P. Cardin, and N. Schaeffer, Turbulent convective length scale in planetary cores, Nature (London) 570, 368 (2019).

[4] G. Budéus, B. Cisewski, S. Ronski, D. Dietrich, and M. Weitere, Structure and effects of a long lived vortex in the Greenland Sea, Geophys. Res. Lett 31, L05304 (2004).

[5] R. P. J. Kunnen, The geostrophic regime of rapidly rotating turbulent convection, J. Turbul. 22, 267 (2021).

[6] R. P. J. Kunnen, B. J. Geurts, and H. J. H. Clercx, Experimental and numerical investigation of turbulent convection in a rotating cylinder, J. Fluid Mech. 642, 445 (2010). 
[7] G. Ahlers, E. Bodenschatz, D. Funfschilling, S. Grossmann, X. He, D. Lohse, R. J. A. M. Stevens, and R. Verzicco, Logarithmic Temperature Profiles in Turbulent Rayleigh-Bénard Convection, Phys. Rev. Lett. 109, 114501 (2012).

[8] R. du Puits, C. Resagk, and A. Thess, Thermal boundary layers in turbulent Rayleigh-Bénard convection at aspect ratios between 1 and 9, New J. Phys. 15, 013040 (2013).

[9] R. P. J. Kunnen, Y. Corre, and H. J. H. Clercx, Vortex plume distribution in confined turbulent rotating convection, Europhys. Lett. 104, 54002 (2013).

[10] J. S. Cheng, S. Stellmach, A. Ribeiro, A. Grannan, E. M. King, and J. M. Aurnou, Laboratory-numerical models of rapidly rotating convection in planetary cores, Geophys. J. Int. 201, 1 (2015).

[11] H. Rajaei, R. P. J. Kunnen, and H. J. H. Clercx, Exploring the geostrophic regime of rapidly rotating convection with experiments, Phys. Fluids 29, 045105 (2017).

[12] H. Rajaei, K. M. J. Alards, R. P. J. Kunnen, and H. J. H. Clercx, Velocity and acceleration statistics in rapidly rotating Rayleigh-Bénard convection, J. Fluid Mech. 857, 374 (2018).

[13] J. S. Cheng, M. Madonia, A. J. Aguirre Guzmán, and R. P. J. Kunnen, Laboratory exploration of heat transfer regimes in rapidly rotating turbulent convection, Phys. Rev. Fluids 5, 113501 (2020).

[14] K. Julien, A. M. Rubio, I. Grooms, and E. Knobloch, Statistical and physical balances in low Rossby number Rayleigh-Bénard convection, Geophys. Astrophys. Fluid Dyn. 106, 392 (2012).

[15] R. P. J. Kunnen, R. Ostilla-Mónico, E. P. van der Poel, R. Verzicco, and D. Lohse, Transition to geostrophic convection: The role of the boundary conditions, J. Fluid Mech. 799, 413 (2016).

[16] H. P. Greenspan, The Theory of Rotating Fluids (Cambridge University Press, Cambridge, 1968).

[17] A. J. Aguirre Guzmán, M. Madonia, J. S. Cheng, R. Ostilla-Mónico, H. J. H. Clercx, and R. P. J. Kunnen, Force balance in rapidly rotating Rayleigh-Bénard convection, J. Fluid Mech. 928, A16 (2021).

[18] A. J. Aguirre Guzmán, M. Madonia, J. S. Cheng, R. Ostilla-Mónico, H. J. H. Clercx, and R. P. J. Kunnen, Competition Between Ekman Plumes and Vortex Condensates in Rapidly Rotating Thermal Convection, Phys. Rev. Lett. 125, 214501 (2020).

[19] K. Julien, S. Legg, J. McWilliams, and J. Werne, Rapidly rotating turbulent Rayleigh-Bénard convection, J. Fluid Mech. 322, 243 (1996).

[20] R. P. J. Kunnen, H. J. H. Clercx, and B. J. Geurts, Heat flux intensification by vortical flow localization in rotating convection, Phys. Rev. E 74, 056306 (2006).

[21] R. P. J. Kunnen, B. J. Geurts, and H. J. H. Clercx, Turbulence statistics and energy budget in rotating Rayleigh-Bénard convection, Eur. J. Mech. B Fluids 28, 578 (2009).

[22] F. S. Godeferd and F. Moisy, Structure and dynamics of rotating turbulence: A review of recent experimental and numerical results, Appl. Mech. Rev. 67, 030802 (2015).

[23] S. Chandrasekhar, Hydrodynamic and Hydromagnetic Stability (Oxford University Press, Oxford, 1961).

[24] J. M. Aurnou, V. Bertin, A. M. Grannan, S. Horn, and T. Vogt, Rotating thermal convection in liquid gallium: Multi-modal flow, absent steady columns, J. Fluid Mech. 846, 846 (2018).

[25] W. B. Heard and G. Veronis, Asymptotic treatment of the stability of a rotating layer of fluid with rigid boundaries, Geophys. Fluid Dyn. 2, 299 (1971).

[26] K. Julien, J. M. Aurnou, M. A. Calkins, E. Knobloch, P. Marti, S. Stellmach, and G. M. Vasil, A nonlinear model for rotationally constrained convection with Ekman pumping, J. Fluid Mech. 798, 50 (2016).

[27] R. Verzicco and P. Orlandi, A finite-difference scheme for three-dimensional incompressible flows in cylindrical coordinates, J. Comput. Phys. 123, 402 (1996).

[28] R. Verzicco and R. Camussi, Numerical experiments on strongly turbulent thermal convection in a slender cylindrical cell, J. Fluid Mech. 477, 19 (2003).

[29] R. Ostilla-Mónico, Y. Yang, E. P. van der Poel, D. Lohse, and R. Verzicco, A multiple-resolution strategy for Direct Numerical Simulation of scalar turbulence, J. Comput. Phys. 301, 308 (2015).

[30] A. J. Aguirre Guzmán, Numerical study of rapidly rotating turbulent convection, Ph.D. thesis, Eindhoven University of Technology, 2021.

[31] J. S. Cheng, J. M. Aurnou, K. Julien, and R. P. J. Kunnen, A heuristic framework for next-generation models of geostrophic convective turbulence, Geophys. Astrophys. Fluid Dyn. 112, 277 (2018).

[32] M. Madonia, A. J. Aguirre Guzmán, H. J. H. Clercx, and R. P. J. Kunnen, Velocimetry in rapidly rotating convection: Spatial correlations, flow structures and length scales, Europhys. Lett. 135, 54002 (2021). 
[33] B. I. Shraiman and E. D. Siggia, Heat transport in high-Rayleigh-number convection, Phys. Rev. A 42, 3650 (1990).

[34] J. E. Hart and D. R. Ohlsen, On the thermal offset in turbulent rotating convection, Phys. Fluids 11, 2101 (1999).

[35] K. P. Iyer, J. D. Scheel, J. Schumacher, and K. R. Sreenivasan, Classical 1/3 scaling of convection holds up to $\mathrm{Ra}=10^{15}$, Proc. Natl. Acad. Sci. USA 117, 7594 (2020).

[36] A. Pandey and K. R. Sreenivasan, Convective heat transport in slender cells is close to that in wider cells at high Rayleigh and Prandtl numbers, Europhys. Lett. 135, 24001 (2021).

[37] S. Stellmach, M. Lischper, K. Julien, G. Vasil, J. S. Cheng, A. Ribeiro, E. M. King, and J. M. Aurnou, Approaching the Asymptotic Regime of Rapidly Rotating Convection: Boundary Layers Versus Interior Dynamics, Phys. Rev. Lett. 113, 254501 (2014).

[38] S. Grossmann and D. Lohse, Scaling in thermal convection: A unifying theory, J. Fluid Mech. 407, 27 (2000).

[39] S. Grossmann and D. Lohse, Thermal Convection for Large Prandtl Numbers, Phys. Rev. Lett. 86, 3316 (2001).

[40] S. Grossmann and D. Lohse, Fluctuations in turbulent Rayleigh-Bénard convection: The role of plumes, Phys. Fluids 16, 4462 (2004).

[41] R. J. A. M. Stevens, E. P. van der Poel, S. Grossmann, and D. Lohse, The unifying theory of scaling in thermal convection: The updated prefactors, J. Fluid Mech. 730, 295 (2013).

[42] D. Nieves, A. M. Rubio, and K. Julien, Statistical classification of flow morphology in rapidly rotating Rayleigh-Bénard convection, Phys. Fluids 26, 086602 (2014).

[43] S. Schmitz and A. Tilgner, Heat transport in rotating convection without Ekman layers, Phys. Rev. E 80, 015305(R) (2009).

[44] S. Schmitz and A. Tilgner, Transitions in turbulent rotating Rayleigh-Bénard convection, Geophys. Astrophys. Fluid Dyn. 104, 481 (2010).

[45] M. Plumley, K. Julien, P. Marti, and S. Stellmach, The effects of Ekman pumping on quasi-geostrophic Rayleigh-Bénard convection, J. Fluid Mech. 803, 51 (2016).

[46] M. Plumley, K. Julien, P. Marti, and S. Stellmach, Sensitivity of rapidly rotating Rayleigh-Bénard convection to Ekman pumping, Phys. Rev. Fluids 2, 094801 (2017).

[47] C. Guervilly, D. W. Hughes, and C. A. Jones, Large-scale vortices in rapidly rotating Rayleigh-Bénard convection, J. Fluid Mech. 758, 407 (2014).

[48] S. Maffei, M. J. Krouss, K. Julien, and M. A. Calkins, On the inverse cascade and flow speed scaling behaviour in rapidly rotating Rayleigh-Bénard convection, J. Fluid Mech. 913, A18 (2021).

[49] E. M. King, S. Stellmach, and B. Buffett, Scaling behaviour in Rayleigh-Bénard convection with and without rotation, J. Fluid Mech. 717, 449 (2013).

[50] S. Sakai, The horizontal scale of rotating convection in the geostrophic regime, J. Fluid Mech. 333, 85 (1997).

[51] I. Grooms, K. Julien, J. B. Weiss, and E. Knobloch, Model of Convective Taylor Columns in Rotating Rayleigh-Bénard Convection, Phys. Rev. Lett. 104, 224501 (2010).

[52] G. K. Batchelor, The Theory of Homogeneous Turbulence (Cambridge University Press, Cambridge, 1953).

[53] M. Wilczek, A. Daitche, and R. Friedrich, On the velocity distribution in homogeneous isotropic turbulence: Correlations and deviations from Gaussianity, J. Fluid Mech. 676, 191 (2011).

[54] M. Buzzicotti, A. Bhatnagar, L. Biferale, A. S. Lanotte, and S. S. Ray, Lagrangian statistics for NavierStokes turbulence under Fourier-mode reduction: Fractal and homogeneous decimations, New J. Phys. 18, 113047 (2016).

[55] M. Wilczek and R. Friedrich, Dynamical origins for non-Gaussian vorticity distributions in turbulent flows, Phys. Rev. E 80, 016316 (2009). 